\title{
Structure and entrainment in the plane of symmetry of a turbulent spot
}

\author{
By BRIAN CANTWELL, DONALD COLES \\ AND PAUL DIMOTAKIS
}

Institute of Technology, Pasadena, California

(Received 31 May 1977 and in revised form 3 October 1977)

Laser-Doppler velocity measurements in water are reported for the flow in the plane of symmetry of a turbulent spot. The unsteady mean flow, defined as an ensemble average, is fitted to a conical growth law by using data at three streamwise stations to determine the virtual origin in $x$ and $t$. The two-dimensional unsteady stream function is expressed as $\psi=U_{\infty}^{2} t g(\xi, \eta)$ in conical similarity co-ordinates $\xi=x / U_{\infty} t$ and $\eta=y / U_{\infty} t$. In these co-ordinates, the equations for the unsteady particle displacements reduce to an autonomous system. This system is integrated graphically to obtain particle trajectories in invariant form. Strong entrainment is found to occur along the outer part of the rear interface and also in front of the spot near the wall. The outer part of the forward interface is passive. In terms of particle trajectories in conical co-ordinates, the main vortex in the spot appears as a stable focus with celerity $0.77 U_{\infty}$. A second stable focus with celerity $0 \cdot 64 U_{\infty}$ also appears near the wall at the rear of the spot.

Some results obtained by flow visualization with a dense, nearly opaque suspension of aluminium flakes are also reported. Photographs of the sublayer flow viewed through a glass wall show the expected longitudinal streaks. These are tentatively interpreted as longitudinal vortices caused by an instability of Taylor-Görtler type in the sublayer.

\section{Introduction}

During the past few years, evidence has been accumulating which suggests that transport in turbulent shear flows is controlled by large coherent flow structures which, although they differ from one type of flow to another, all represent typical and recognizable concentrations of transverse mean vorticity at the largest scale of the flow. In this context a turbulent spot in a laminar boundary layer (Emmons 1951; Schubauer \& Klebanoff 1955) provides an example of an isolated coherent structure which may have important properties in common with structures in a fully developed turbulent boundary layer.

Coles \& Barker (1975, hereafter referred to as CB) took this point of view when making some exploratory measurements of flow in a turbulent spot. They concluded, on the basis of slender and partly circumstantial evidence, that a turbulent spot in a laminar boundary layer at constant pressure is essentially a single large horseshoe vortex structure, that the vortex centre in the plane of symmetry moves at about $0 \cdot 83 U_{\infty}$, and that the spot grows not only by entraining irrotational fluid from the ambient free 
stream but also by entraining rotational fluid from the ambient laminar boundary layer.

An independent and more extensive study by Wygnanski, Sokolov \& Friedman (1976, hereafter referred to as WSF) has established the shape of the turbulent spot in three dimensions and has provided data on all three components of the mean velocity in the interior of the spot. In the plane of symmetry, where the two experiments have some common ground, there is substantial agreement concerning the shape and meanvelocity field. WSF agree with CB that the spot is a large horseshoe vortex, but disagree about certain important properties of the vortex, especially its characteristic speed of propagation. CB deduced a vortex velocity of $0.83 U_{\infty}$ by following a conspicuous minimum in the (ensemble) mean velocity as observed by a fixed probe in the outer part of the flow. WSF proposed as the characteristic spot velocity the celerity deduced in the conventional way from space-time correlations of the streamwise velocity. When they applied this correlation technique very near the wall in the plane of symmetry, they obtained the value $0 \cdot 65 U_{\infty}$.

Both CB and WSF carried out stream-function calculations in moving co-ordinates for the mean flow in the plane of symmetry of the spot. A major issue in these calculations was entrainment, and an obvious objection is that the mean flow was treated as two-dimensional. WSF quieted this objection by showing experimentally that $\partial w / \partial z=0$ near the plane of symmetry and also by showing close agreement between measured and computed values of the normal velocity $v$.

A second and more serious objection is that spot growth was not taken into account. In reality, a typical spot roughly doubles in size during the time interval between the passage of its leading and trailing interfaces past a fixed probe. At best, the stream function $\psi(y, t)$ as calculated from data at one station by CB and by WSF can only suggest the probable form of the instantaneous streamlines. Moreover, the calculated flow pattern is extremely sensitive to the speed chosen for the moving co-ordinate system, and it is precisely this speed which is an important unresolved element in the experiments.

Our primary objective in the present research is to remove this second objection by viewing the process of spot growth in appropriate conical co-ordinates. We describe the experimental means of achieving this end, including an unusual flow-visualization technique, in $\S \S 2-4$. In the main analysis, in $\S \S 5-8$, we establish the conical property for the present data, define a similarity form for $\psi / t$ as a function of $x / t$ and $y / t$, calculate proper instantaneous mean streamlines, and obtain mean particle paths in Galileaninvariant form by integrating the Lagrangian displacement equations graphically. Several singularities revealed by this process are interpreted in terms of structure. In $\$ 9$ the results for particle paths and for the spot shape are combined to determine quantitatively the local rate of entrainment around the spot boundary in the plane of symmetry. Finally, an attempt is made in $\S 10$ to assemble visual information from $\S 3$ and structural information from $\S \S 7-9$ into a coherent kinematic description of the turbulent spot. 


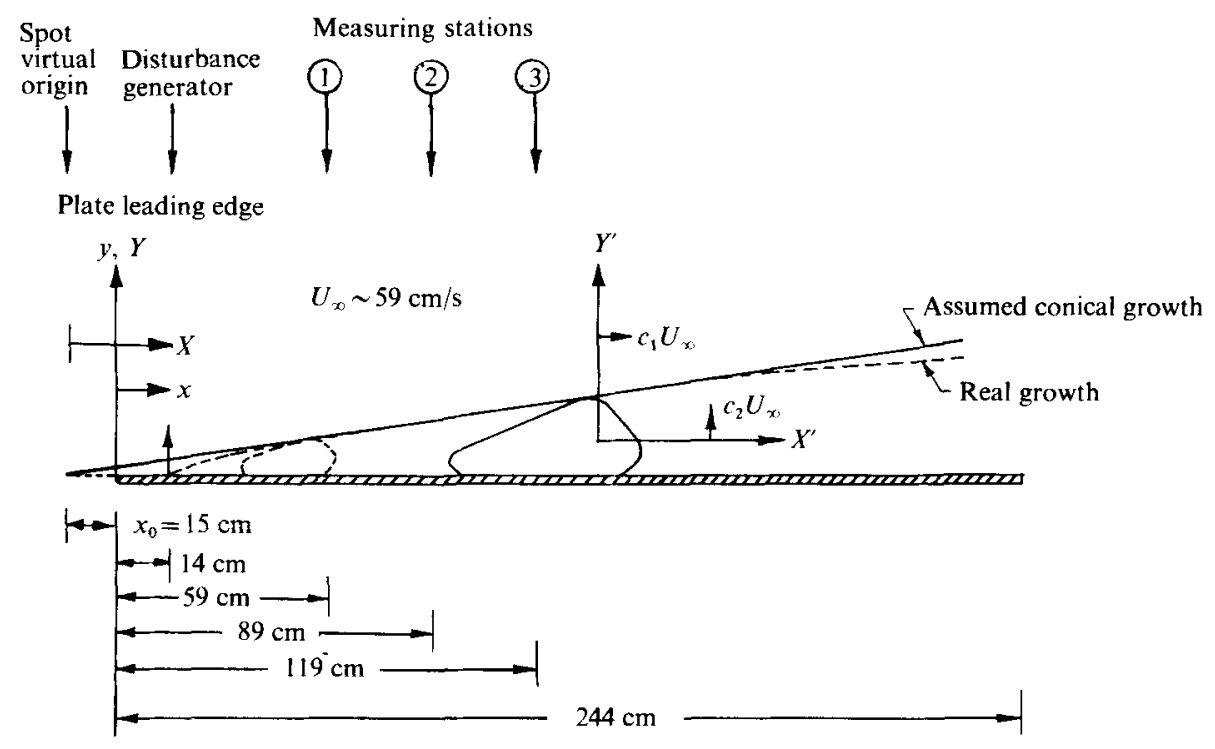

FiguRE 1. Sketch of flat-plate model, showing important dimensions and co-ordinate systems.

\section{Model and test conditions}

The experiment was carried out in the GALCIT $\dagger$ low-speed water channel, which has a working section $5.8 \mathrm{~m}$ long, $46 \mathrm{~cm}$ wide and $61 \mathrm{~cm}$ deep. The flat-plate model, sketched in figure 1, was the Plexiglas plate used by CB. Several changes were made in the model and in the test conditions to suit the purposes of the present research. The stream speed was approximately doubled, to about $59 \mathrm{~cm} / \mathrm{s}$, in order to obtain greater separation between the sublayer scale and overall scale of the flow. The orifice for the jet disturbance was moved upstream to a point $14 \mathrm{~cm}$ from the plate leading edge. The disturbance generator was a small three-roller peristaltic pump conneeted to a constantspeed motor through a magnetic-particle clutch. By energizing this clutch for a short time, the pump could be rotated rapidly through a third of a revolution on command. One other structural change made in the model was the installation of several transverse cables on the underside of the plate about $2 \mathrm{~cm}$ from the plate surface. Tension was applied to these cables to bow the top or working surface into a slightly convex shape. After this change, laser-Doppler measurements could be made as close to the wall as $0 \cdot 013 \mathrm{~cm}$.

The leading edge of the model was set appreciably lower than the trailing edge in an effort to prevent premature natural transition by placing the forward stagnation line above the centre of the elliptical nose. The depth of the model below the free surface therefore decreased in the direction of flow while the measured velocity in the free stream increased, as shown in figure 2 . The product of depth and velocity increased slightly, presumably because of displacement effects from boundary layers on the various walls.

† Graduate Aeronautical Laboratories, California Institute of Technology. 


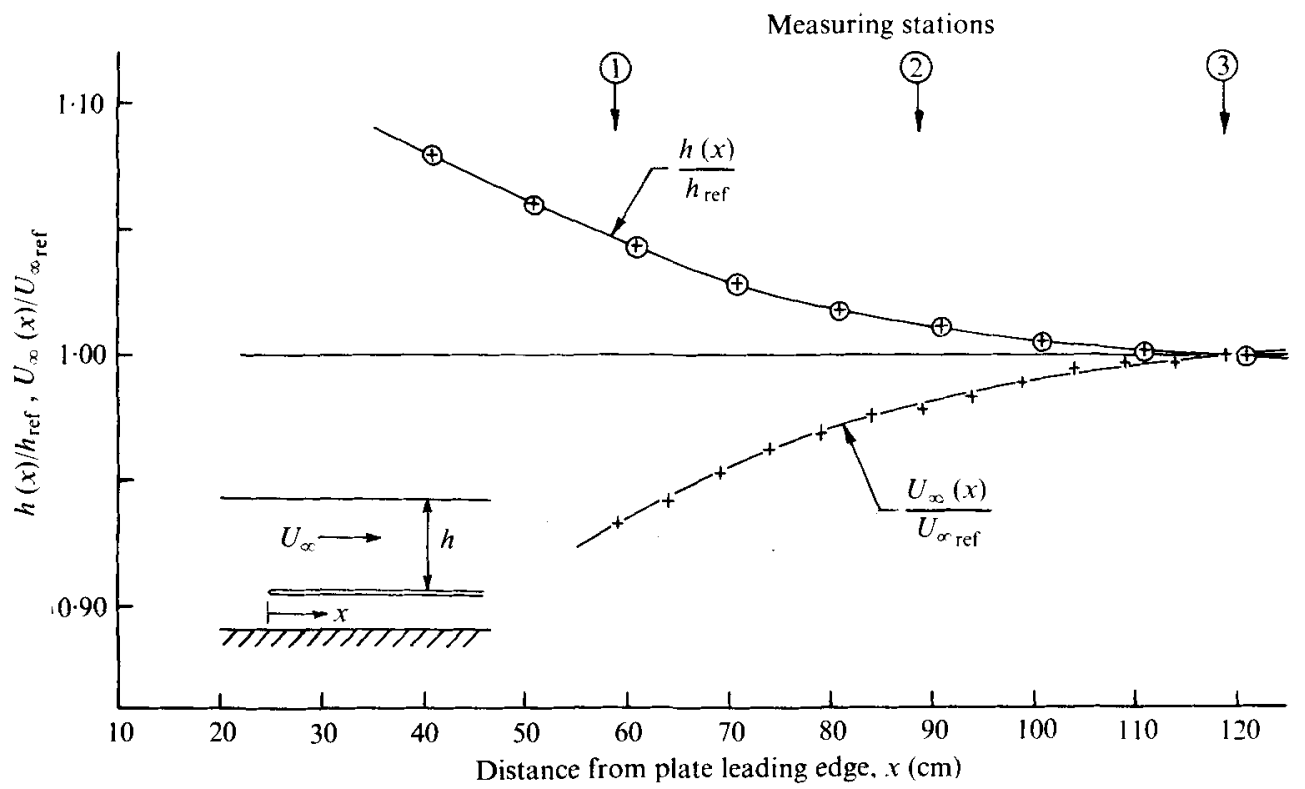

Frgure 2. Measured variation of water depth $h$ and free-stream velocity $U_{\infty}$ along plate.

Reference values at third measuring station: $h_{\text {ref }}=12 \cdot 1 \mathrm{~cm} ; U_{\text {oret }}=59 \cdot 5 \mathrm{~cm} / \mathrm{s}$.

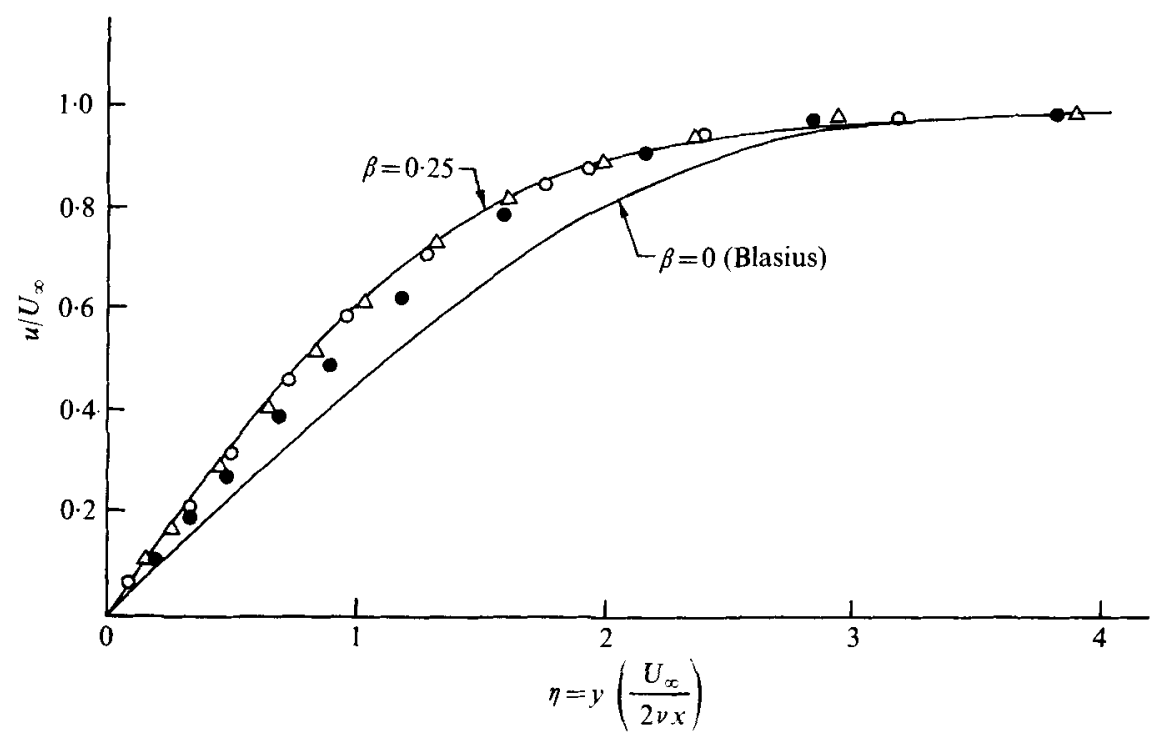

Figure 3. Measured laminar velocity profiles at three measuring stations. $\triangle, x=59 \mathrm{~cm}$; $O, x=89 \mathrm{~cm} ; O, x=119 \mathrm{~cm}$. Computed profiles are from Smith $(1954) ; \beta$ is the FalknerSkan parameter.

Although the undisturbed boundary layer in the plane of symmetry remained laminar almost to the trailing edge, transverse contamination from the side walls began to interfere with spot growth about half way along the plate. The three main series of measurements were therefore made at stations located 59,89 and $119 \mathrm{~cm}$ from the leading edge, as indicated in figure 1. The Reynolds number at the third station, 
based on the local free-stream velocity of $59.5 \mathrm{~cm} / \mathrm{s}$ and the distance from the leading edge, was about $0.82 \times 10^{6}$.

Velocity profiles obtained in the undisturbed laminar boundary layer are shown in figure 3 in Blasius co-ordinates. The effect of free-stream acceleration in thinning the laminar layer is apparent. If the early part of the distribution $U_{\infty}(x)$ in figure 2 is fitted by a power law $U_{\infty} \sim x^{m}$, the exponent $m$ is found to be about $0 \cdot 14$. The corresponding Falkner-Skan parameter $\beta=2 m /(m+1)$ is about $0 \cdot 25$. The profile for this value of $\beta$ (Smith 1954) is included in figure 3 and is reasonably close to the measurements at the first two stations. The measurements at the third station are well represented by the Blasius profile if the boundary-layer thickness is taken to be about $20 \%$ less than the thickness calculated for $d p / d x=0$.

\section{Flow visualization}

Two different flow-visualization techniques were used to support the measurements reported here. The first technique was dye visualization like that applied to the spot problem by Elder (1960) and by Meyer \& Kline (1961). In the present instance, a thin layer of dyed fluid was laid down very near the plate surface through a slot about $10 \mathrm{~cm}$ from the leading edge. CB used potassium permanganate dye in this way, but did not take photographs. Coles later obtained a motion-picture record of both the spot and the synthetic boundary layer. A similar record was made during the present experiments, except that the dye was vegetable food colouring. Two frames from these motion pictures showing the spot shape in plan view are reproduced in figures $4(a)$ and $(b)$ (plate 1). Motion pictures of a turbulent spot in air have also been made by M. R. Head at Cambridge, using smoke in the laminar boundary layer for visualization. By kind permission of Dr Head, two frames from his motion pictures are reproduced in figures $5(a)$ and $(b)$ (plate 2). As far as we are aware, none of these observers of the turbulent spot has reported seeing anything like the splitting which is known to occur under certain conditions for the puff in pipe flow. Each spot seems to remain intact and recognizable for as long as it can be seen. $f$

Dye (or smoke) visualization is particularly effective in showing linear growth because the spot entrains dyed fluid from the wall region, picking it up like a vacuum cleaner. This dyed fluid is rapidly broken up and dispersed throughout the body of the spot before there is appreciable dilution. If the plate surface is initially completely covered with dye, entrainment is seen to occur along the full span of the spot. To the rear, the spot leaves behind a wedge-shaped wake of nearly clear fluid. This wake is slowly erased by downstream motion of the adjacent dye. When a second spot follows closely behind the first one, entrainment is visible only at the lateral extremities of the spot (of. figure $4 b$, plate 1). Dye which is present near the plane of symmetry of the second spot must therefore have been picked up at a much earlier time. This dye tends to be more concentrated near the leading edge of the spot as the spot moves downstream. Another observation, also illustrated by figure $4(b)$, is that a region of trans-

$\dagger$ Wygnanski, Haritonidis \& Kaplan (1978) have recently reported some remarkable observations of regular wave packets at the outer edges of the laminar wake of a spot. These wave packets eventually degenerate into new turbulence. We have found no evidence of this phenomenon in our motion pictures, perhaps because our Reynolds numbers were not sufficiently large. 
verse contamination may be constructed of isolated structures which resemble half-spots.

The second flow-visualization technique was less conventional. It employed an extremely dense suspension of aluminium flakes in the channel (about one part in three hundred by weight). Even under strong illumination, it was not possible to see more than 2 or $3 \mathrm{~mm}$ into the fluid. The suspension was prepared by wetting a quantity of dry powder (Alcan, grade M. D. 7300) with a liquid detergent to obtain a paste which was then diluted with water until it flowed freely. Any unwetted flakes were skimmed off and the remaining mixture was poured into the channel while stirring continuously. In this way about $15 \mathrm{~kg}$ of aluminium was added to the $4500 \mathrm{l}$ of water in the channel. No attempt was made to establish whether or not the final suspension behaved like a Newtonian fluid. The aluminium flakes were about $0.2 \mu \mathrm{m}$ in thickness and their largest dimension ranged from perhaps $5 \mu \mathrm{m}$ to as much as $30 \mu \mathrm{m}$. They tended to settle slowly on the channel bottom, which therefore had to be swept or hosed at intervals.

Although there is no quantitative theory for the action of the flakes in changing the reflective properties of the suspension, it is certain that the flakes are oriented in a systematic way by the three-dimensional rate-of-strain field in the fluid. Moreover, they respond very rapidly to changes in this field. Information contained in a single photograph is essentially iniormation about the current state of the motion. This is an entirely different basis for flow visualization from that provided by smoke, dye, bubbles or other passive tracers. A single photograph using such tracers shows the cumulative result of all the various processes which have transported and concentrated or dispersed the tracer material, whether or not these processes are still active.

In order to use this aluminium visualization technique effectively, the flat-plate model was removed and a stable laminar boundary layer was established on the glass side walls of the channel. Spots were generated by touching the wall momentarily with a small rod. Two photographs obtained using this technique are reproduced in figures $6(a)$ and $(b)$ (plate 3 ). The depth of view into the fluid is always less than or at most comparable to the scale of the smallest visible disturbances. In regions where the flow is turbulent, in particular, the photographs show only the motion in the lower part of the sublayer. Figure $7(a)$ (plate 4 ) is unique in showing a half-spot, generated by touching the wall at the free surface. The photograph is a view of the channel side wall, as usual, but a mirror has been mounted above the channel at $45^{\circ}$ to show also a crosssection through the spot as seen at the free surface. Figure $7(b)$ (plate 4 ) is a similar view of a fully turbulent boundary layer, with transition induced by a trip wire near the entrance to the channel. A stationary motion-picture camera was used to photograph a variety of spot patterns, mostly of the kind shown in figure 6 . A motion-picture record was also made of the flow at the free surface for the case of the half-spot. For the latter purpose the camera was mounted above the channel on a carriage and was traversed manually to follow the spot.

There is no doubt that the photographs in figures 6 and 7 show a new aspect of the sublayer streaks first observed at Stanford. Meyer \& Kline (1961) found these streaks to be present under a turbulent spot, a turbulent wedge and a turbulent boundary layer. Our photographs confirm their conclusion and add some new information. The visualization of sublayer flow by the aluminium technique in figures 6 and 7 shows at first sight a confused, knotted structure which on eloser examination is seen to be highly elongated in the stream direction. Individual streaks can often be followed for 


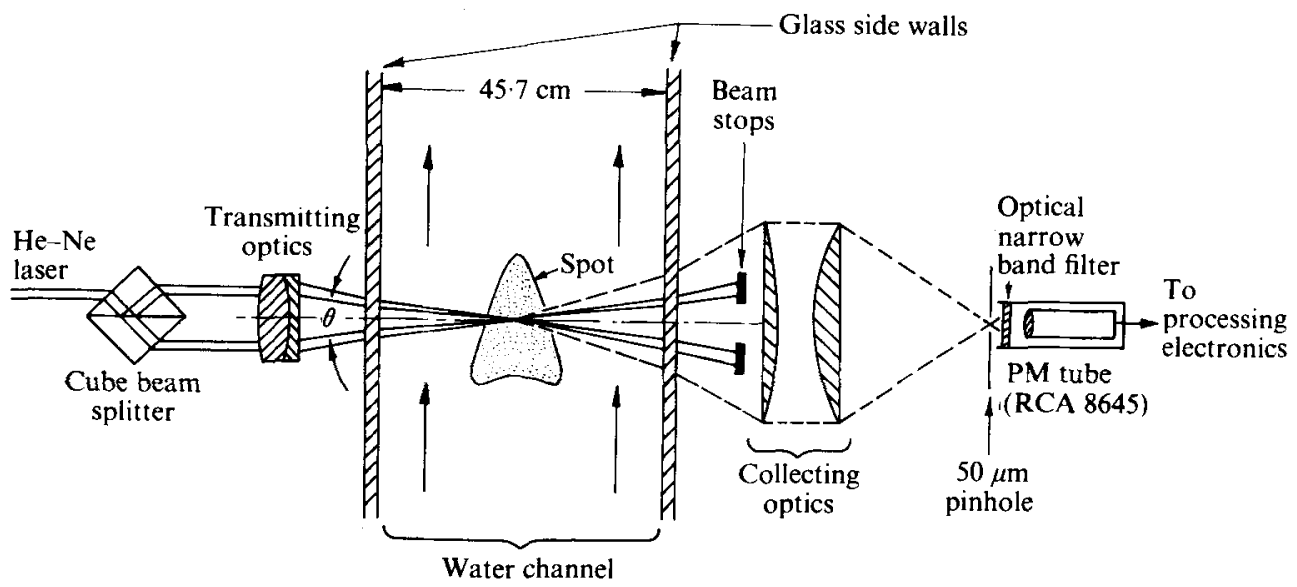

FIGURE 8. Arrangement of transmitting and receiving optics for laser-Doppler velocimetry (not drawn to scale).

long distances. Quantitative evidence was obtained by using an optical correlation technique (Kovasznay 1949) to measure the scale $\lambda$ in the cross-flow direction for the boundary-layer flow in figure $7(b)$. In dimensionless form, the value obtained $\dagger$ is $\lambda^{+}=u_{\tau} \lambda / \nu=86$, close enough to the generally accepted value $\lambda^{+} \sim 100$.

\section{Velocity measurements}

The streamwise velocity was measured using single-particle laser-Doppler velocimetry in the dual-scatter mode. The optical arrangement is sketched in figure 8 . The helium-neon laser (Spectra Physies, model 120) had a power output of $5 \mathrm{~mW}$. A 50-50 cube beam splitter was used to generate the two scattering beams and was carefully adjusted to match the focusing and crossing points of the two beams within the water channel. The angle between the two beams in air was measured as $4 \cdot 18^{\circ}$, giving a fringe spacing of $\frac{1}{2} \lambda / \sin \frac{1}{2} \theta=8.68 \mu \mathrm{m}$ and a conversion factor of $1.15 \mathrm{kHz}(\mathrm{cm} / \mathrm{s})^{-1}$. Approximately 16 detectable fringes were present in the focal volume.

With the focal volume at the mid-span, traverses were made normal to the plate surface at three stations downstream of the disturbance generator (cf. figure 1). Each signal burst arising from passage of a single scattering particle through the focal volume was processed to extract the time of flight for four fringe-plane intervals $(34 \cdot 7 \mu \mathrm{m})$. This choice for the number of intervals avoided any undue restriction on the direction of the measurable velocity vectors (Dimotakis 1976). The time of flight was determined with a resolution of $1 \mu \mathrm{s}$ using a $1 \mathrm{MHz}$ time base derived from a $100 \mathrm{MHz}$ crystal-controlled master oscillator.

The real time of each signal burst was also measured by reading a free-running counter at the time of the first fringe crossing acceptable to the electronics. The real time was determined with a resolution of $1 \mathrm{~ms}$ using a $1 \mathrm{kHz}$ time base derived from

$\dagger$ The measured value of $\lambda$ was $1.06 \mathrm{~cm}$. The value obtained for the stream velocity $U_{\infty}$ by timing a small float was $15.0 \mathrm{~cm} / \mathrm{s}$. The viscosity $\nu$ was $0.0087 \mathrm{~cm}^{2} / \mathrm{s}$. The boundary-layer thickness $\delta$ as viewed in the free surface in figure $7(b)$ was estimated to be about $6 \cdot 1 \mathrm{~cm}$. The correlation by Coles (1962) was used to relate $U_{\infty} \delta / \nu$ to $u_{\tau} / U_{\infty}=\left(\frac{1}{2} C_{f}\right)^{\frac{1}{2}}$. 


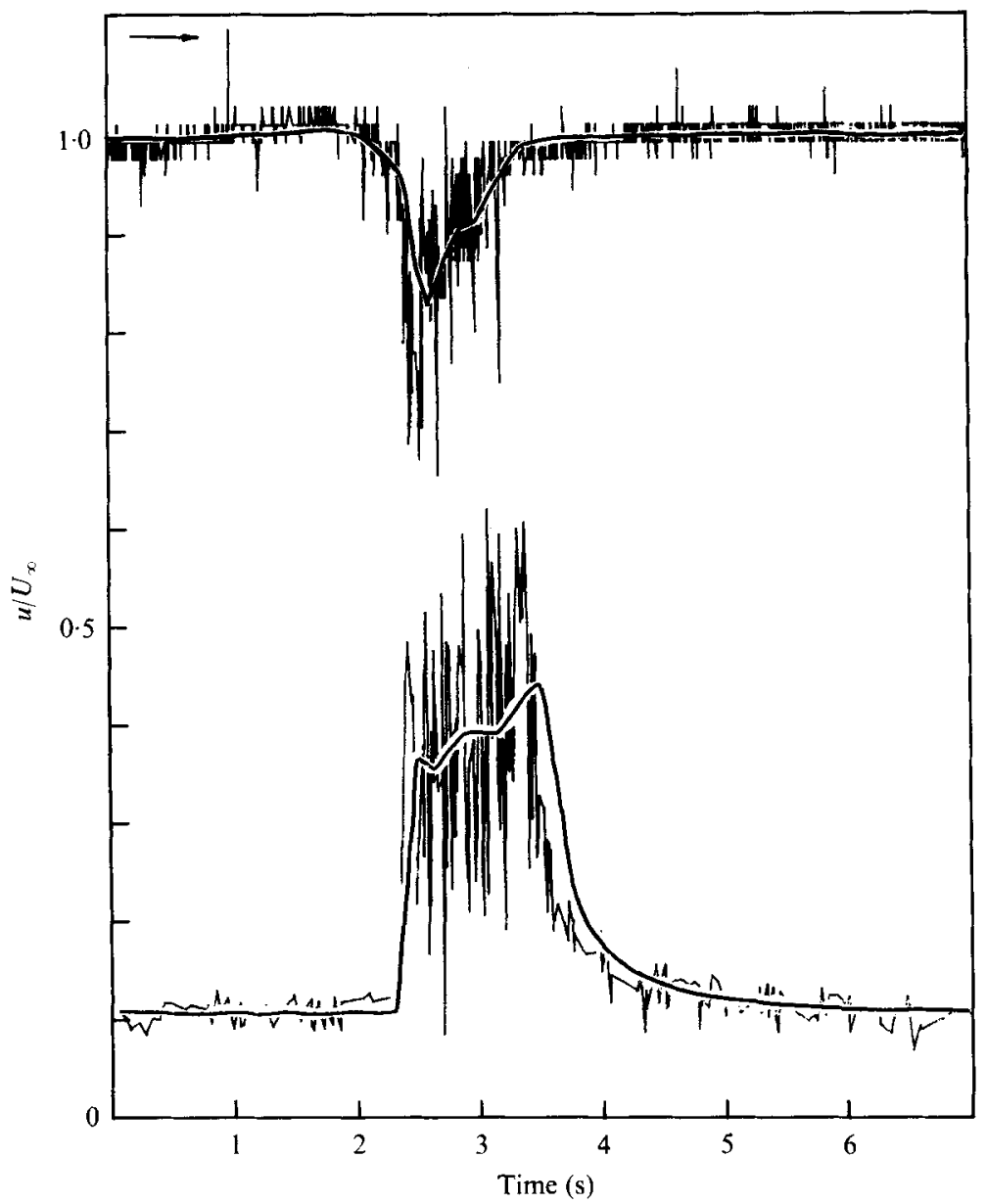

Froure 9. Typical velocity traces for single realization and for ensemble-mean flow at third measuring station. Centre of focal volume is at $y=0.038 \mathrm{~cm}$ (lower traces) or $y=0.953 \mathrm{~cm}$ (upper traces).

the same $100 \mathrm{MHz}$ master oscillator. A spot was generated once every $7 \cdot 22 \mathrm{~s}$ by a trigger pulse which also reset the real-time counter to zero.

Figure 9 shows two typical data traces for a single spot passing the third measuring station, with the focal volume centred at $y=0.038 \mathrm{~cm}$ (lower trace) and at $y=0.953 \mathrm{~cm}$ (upper trace). The individual data points are connected by straight-line segments to simulate continuous high-frequency traces. The spot is generated at $t=0$ and arrives at the measuring station about $2 \mathrm{~s}$ later. In the upper trace, the fluctuations before and after passage of the spot are not caused by free-stream turbulence, but by low resolution in the time-of-flight measurement. The fluctuations in the lower trace have a different cause; this is the presence of a substantial velocity gradient in the steady laminar boundary layer together with the finite extent $(0.012 \mathrm{~cm})$ of the focal volume in the direction normal to the wall (Kreid 1974; Mayo 1974). Note the very rapid increase in velocity close to the wall when the spot arrives. In the example shown in the lower trace in figure 9 , the velocity increases by a factor of five in the time interval between the passage of two consecutive scattering particles through the focal volume. There is also 


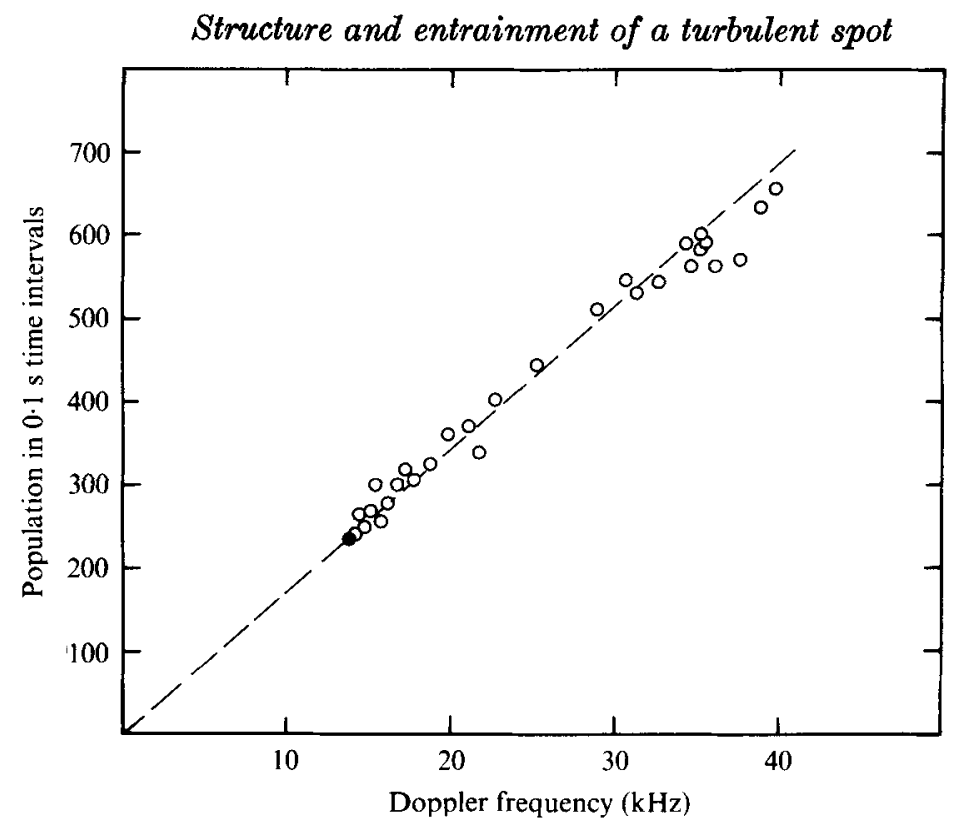

Figure 10. Sampling frequency during spot passage as a function of harmonic-mean Doppler frequency. Both variables are ensemble averages for about 40 spots. Centre of focal volume is at $x=119 \mathrm{~cm}, y=0.064 \mathrm{~cm}$.

a very high fluctuation level inside the spot, with instantaneous velocities ranging from $0 \cdot 1 U_{\infty}$ to $0 \cdot 6 U_{\infty}$.

During the experiment, each data run at a given $x, y$ location continued until a specified number of particles had been counted. This number was normally chosen to correspond to a population of 30-60 spots. During data processing, individual velocity measurements were first sorted according to their real time into $0 \cdot 1 \mathrm{~s}$ time intervals, starting from $t=0$. A typical population for each time interval was several hundred measurements. 'To represent this population systematically as a velocity histogram, the minimum and maximum measured values of the Doppler frequency for the given time interval were truncated and rounded up, respectively, until their integer difference (in units of $10 \mathrm{~Hz}$ ) was divisible by 24 . The frequency data within each time interval were then sorted into a histogram of 24 bins spanning the observed range of frequencies.

Two editing operations were carried out on the raw data. The LDV processor was able to record data and reset itself for the next reading in less than $1 \mu \mathrm{s}$. Because of the small number of fringe crossings required for a measurement, multiple measurements were sometimes made for the same particle as it traversed the focal volume. These were identified by their coincidence in real time and were averaged to obtain one velocity measurement per particle. Although the LDV processor performed time and amplitude tests on every cycle of each signal burst (Dimotakis \& Lang 1974), it was possible to obtain an occasional erroneous measurement because of the finite signal-to-noise ratio or failure to reject signal bursts arising from multiple-particle scattering. One such measurement is indicated by the arrow near the top trace in figure 9. The method used to remove such obviously erroneous measurements was to compute the mean and the r.m.s. deviation $\sigma$ for the frequency data of each histogram. Points falling outside a tolerance of $4 \sigma$ on either side of the mean were rejected. This process was carried out twice for each histogram to obtain the final data used to define the mean velocity. No 

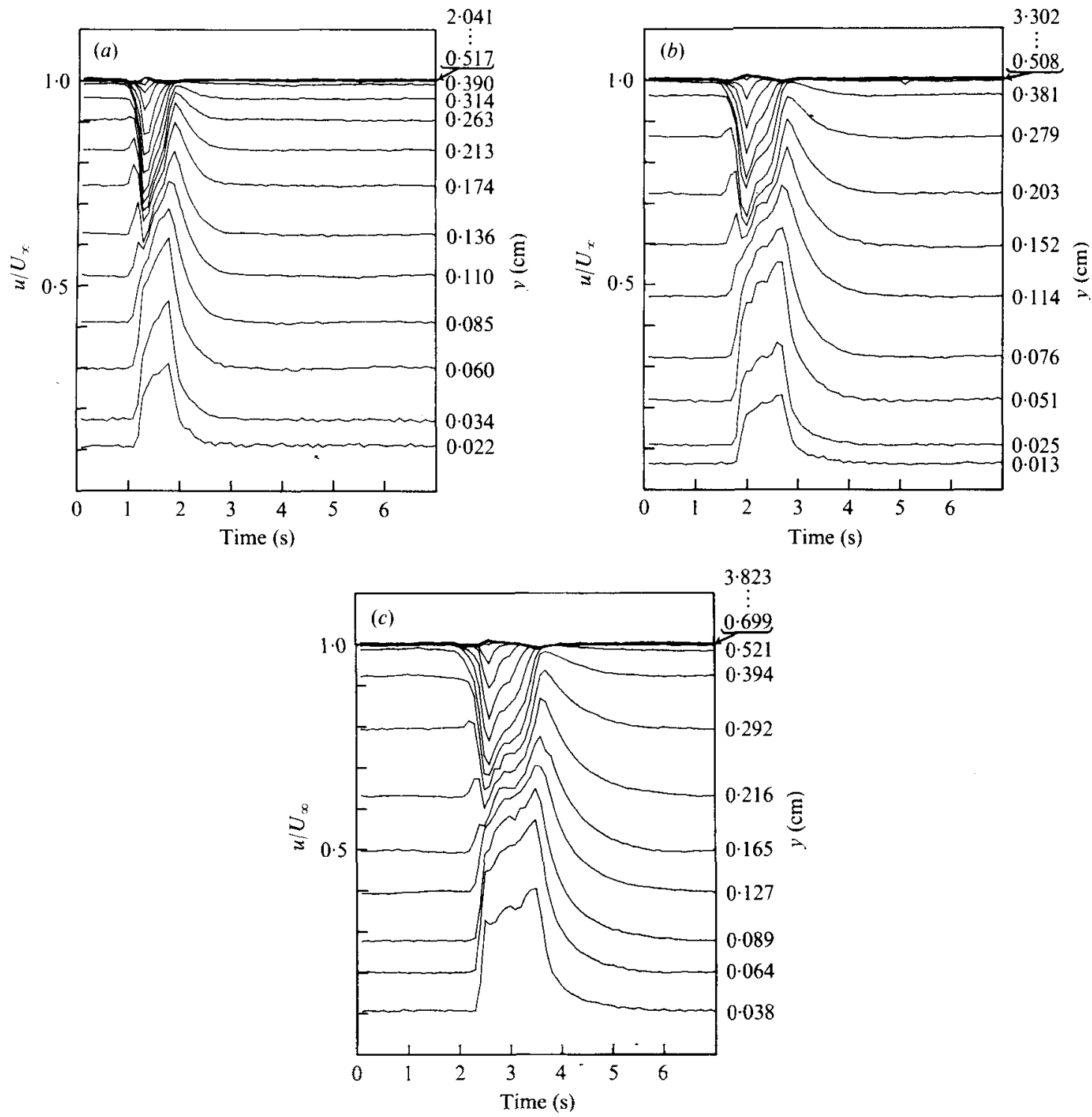

FIGURE 11. Basic data for experiment. Ensemble-mean velocity as a function of $t$ for various values of $y$. (a) $x=59 \mathrm{~cm}$. (b) $x=89 \mathrm{~cm}$. (c) $x=119 \mathrm{~cm}$.

more than a few dozen measurements had to be discarded out of a total of several million.

Evaluation of the mean velocity from single-particle LDV data is subject to a sampling bias caused by the fact that higher velocities are observed more often (McLaughlin \& Tiederman 1973). The sampling bias can be computed as a function of the velocity vector $\mathbf{u}$, the angle $\theta$ between the two beams and the ratio $\epsilon$ of the minimum number of fringe crossings specified in the electronics to the total number of fringes in the focal volume (Dimotakis 1976). If $\epsilon^{2}, \sin ^{2} \frac{1}{2} \theta$ and $v^{2} / u^{2}$ are all much less than unity ( $\epsilon \sim 0.25$ for the present experiment), the sampling bias can be shown to be directly proportional to $u$, as originally proposed by McLaughlin \& Tiederman. An unbiased 
estimate of the mean velocity can then be obtained in terms of the harmonic mean of the individual measurements, i.e.

$$
\frac{1}{u} \simeq \frac{1}{N} \sum_{i=1}^{N} \frac{1}{u_{i}} \simeq \frac{1}{N} \sum_{k=1}^{24} \frac{n_{k}}{u_{k}}
$$

where the second form refers specifically to a velocity histogram, $N$ being the total population and $n_{k}$ the population of the $k$ th velocity bin centred at $u_{k}$. The relatively smooth traces in figure 9 are made up of straight-line segments connecting such harmonic means, with the data centred in successive $0.1 \mathrm{~s}$ intervals in time.

To illustrate the validity of the bias-correction algorithm, data obtained at $x=119 \mathrm{~cm}$ and $y=0.064 \mathrm{~cm}$ are shown in figure 10 . The abscissa is the corrected (harmonic mean) Doppler frequency corresponding to a particular time interval. The ordinate is the associated population. The approximation of a linear relation appears to be quite adequate. In the present measurements, the difference between the biased mean velocity (ensemble average) and harmonic mean velocity ranged from a fraction of $1 \%$ to as much as $20 \%$. The largest differences occurred close to the wall near the leading edge of the spot, where dispersion in the spot arrival time resulted in a mixture of laminar and turbulent samples.

It is worth noting that use of the harmonic mean also corrects, at least for laminar flow, the velocity-gradient bias which is caused by the finite vertical extent of the focal volume (Kried 1974; Mayo 1974). The filled symbol nearest the origin in figure 10 corresponds, in fact, to the laminar data.

Mean velocities observed at the three measuring stations at $x=59,89$ and $119 \mathrm{~cm}$ are plotted as a function of $t$ for various values of $y$ in figure 11. These data are the basis of the analysis which follows. They have been filed in tabulated form in the aeronautics library at GALCIT and can be obtained on application to the librarian.

\section{The conical property}

Figure 12 is a detail of four typical records of mean velocity against time at the third measuring station. For convenience, we have chosen to represent each record by several straight-line segments which intersect at the points labelled $A, \ldots, G$ in the figure. Point $A$ marks the first occurrence of a detectable change in velocity. Point $C$ marks the main velocity minimum. Point $F$ marks either the final velocity maximum (close to the wall) or the last substantial disturbance (far from the wall). At $G$ the relaxation from the velocity maximum at $F$ to the final laminar velocity is $90 \%$ complete (the relaxation is of course detectable up to later times). The three points $B, D$ and $E$ are well defined, but their meaning is not, so they are simply carried along without prejudice in what follows.

When all the data are treated in this way, the intersections $A, \ldots, G$ define several characteristic loci in the eross-section of the spot, as indicated in figure 13. The first use of these loci is to test the conical growth property for the spot in terms of $x$ and $t$ only. Each locus is represented by its earliest or latest time of arrival at a given station, as appropriate, and a set of rays is drawn through the corresponding arrival times. To good accuracy, the virtual origin of the spot in $x$ can be placed $29 \mathrm{~cm}$ upstream of the disturbance orifice, or $15 \mathrm{~cm}$ upstream of the leading edge of the plate, while the virtual origin in $t$ can be placed $0.34 \mathrm{~s}$ earlier in time than the actual disturbance pulse. Note 


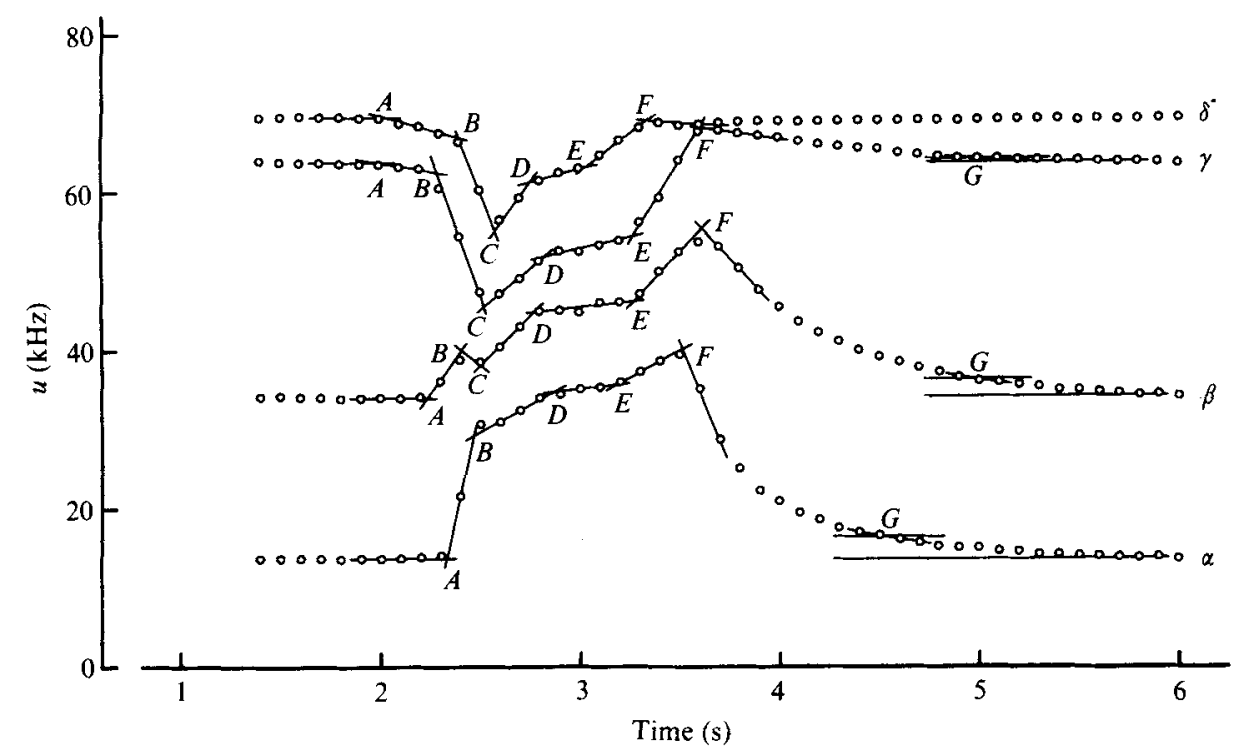

FIGURE 12. Four typical traces of ensemble-mean velocity at third measuring station, showing method used to define loci $A, \ldots, G$. Distance from wall $\alpha, 0.064 \mathrm{~cm} ; \beta, 0.165 \mathrm{~cm} ; \gamma, 0.394 \mathrm{~cm}$; $\delta, 0.953 \mathrm{~cm}$.

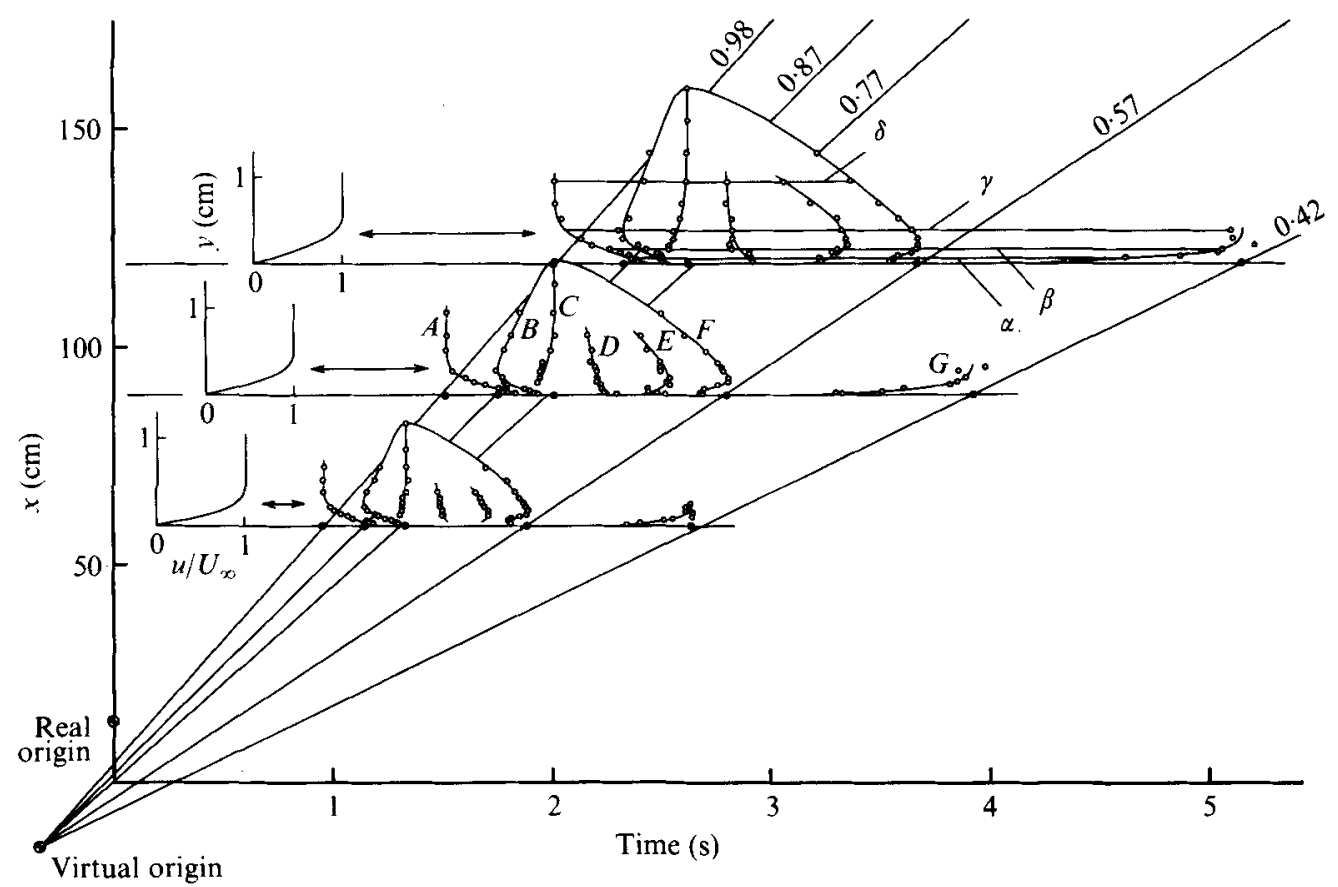

Figure 13. Construction to locate virtual origin at $x_{0}=-15 \mathrm{~cm}, t_{0}=-0.34 \mathrm{~s}$ for best approximation to conical growth. See figure 12 for mean-velocity traces along horizontal lines labelled $\alpha, \beta, \gamma, \delta$ at third measuring station. 


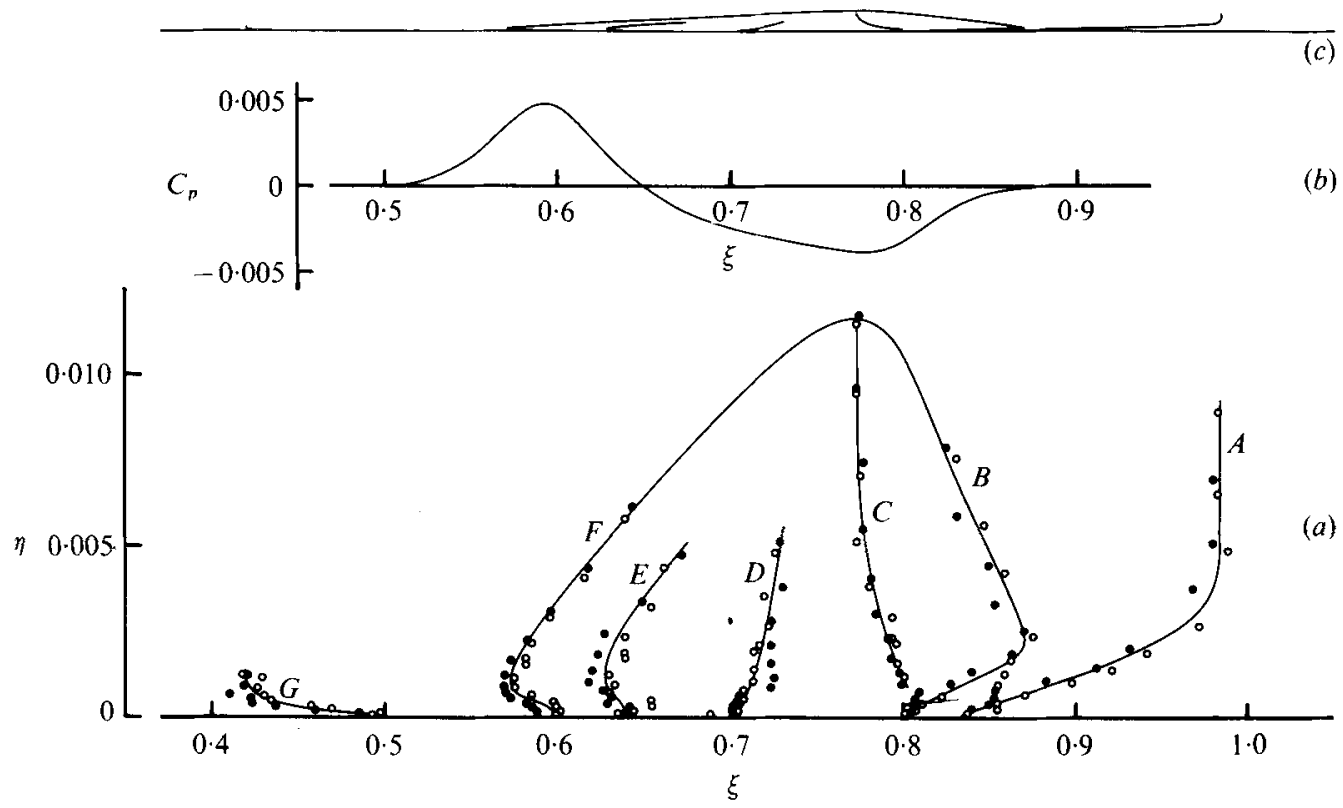

Fredre 14. (a) Superposition of loci $A, \ldots, G$ in conical co-ordinates $(\xi, \eta) . \bigcirc, X=104 \mathrm{~cm}$; , $X=134 \mathrm{~cm}$. (b) Estimated mean pressure signature at wall. (c) Same as $(a)$ but with identical scales for $\xi$ and $\eta$.

that the variables $x$ and $t$ in figure 13 are directly measured quantities, so that the slight acceleration of the free stream is taken into account to first order in the speed estimates which are included in the figure. The most forward part of the leading locus $B$ has a speed of about $0.87 U_{\infty}$. The most rearward part of the trailing locus $F$ has a speed of about $0.57 U_{\infty}$. The velocity minimum on locus $C$ has a speed of about $0 \cdot 80 U_{\infty}$ near the wall and a speed of about $0.77 U_{\infty}$ near the mid-height in the spot, rather than $0 \cdot 83 U_{\infty}$ as reported by $\mathrm{CB}$.

The conical property may now be further tested in terms of $x, y$ and $t$ by superposing the various loci in co-ordinates

$$
\xi=X / U_{\infty} T, \quad \eta=Y / U_{\infty} T,
$$

where $X=x-x_{0}$ and $T=t-t_{0}$ are measured from their respective virtual origins as just defined (i.e. $x_{0}=-15 \mathrm{~cm}, t_{0}=-0.34 \mathrm{~s}$ ). The result is shown in figure 14 . For heuristic purposes, and for the limited range of the present experiments, it is clearly permissible to treat the growth as conical in both $x$ and $y \cdot \dagger$

Figure 14 is a proper profile of a spot, unlike the profiles in figure 13 , which were obtained from observations at a fixed station. Such observations measure the flow field at the front of the spot when the spot is small, but measure the flow field at the rear when the spot is considerably larger. To call attention to the real scales of the motion, the same proper profile is shown undistorted (i.e. with the same scale for $\xi$ and $\eta$ ) at the top of figure 14 .

$\dagger$ The virtual origin for $z$, as judged from the observed rate of growth in the motion-picture record represented by figure $4(b)$, may be closer to the disturbance source. 
As an operational definition, we now take the active region in a turbulent spot to be the region inside the closed contour $B F$ in figures 13 and 14 . This active region is preceded and followed by regions $A B$ and $F G$, respectively, in which there is an accommodation to an environment which consists in part of a uniform flow and in part of a laminar boundary layer. In this respect our picture of the spot is conceptually quite different from previously published pictures (Schubauer \& Klebanoff 1955; CB; WSF). These authors have defined the spot boundary in terms of a threshold perturbation in the mean velocity or in terms of a threshold turbulence intensity, and thus may have included all or part of the accommodation regions $A B$ and $F G$ within the body of the spot.

One immediate application of the conical property is estimation of the pressure signature at the surface. Figures $11(a)-(c)$ all show weak velocity perturbations in the potential flow in the free stream during the passage of the spot. Let $u_{\infty}(t)$ and $p(t)$ be the perturbed velocity and pressure and let $U_{\infty}$ and $P$ be the steady values at much earlier or much later times. To a useful approximation, the flow in the free stream satisfies the linearized unsteady Bernoulli equation

$$
(p-P) / \rho=U_{\infty}\left(U_{\infty}-u_{\infty}\right)-\partial \phi / \partial t
$$

where $\phi$ is the velocity potential and in general $u=\partial \phi / \partial x$. Conical similarity requires $u_{\infty} / U_{\infty}$ to have the form $1+f^{\prime}(\xi)$, where $f^{\prime}=d f / d \xi$. Integration with respect to $x$ at constant $t$ gives

$$
\phi / U_{\infty}^{2} T=\xi+f(\xi)
$$

Differentiation with respect to $t$ and substitution in (3) yield finally

$$
C_{p}=(p-P) /\left(\frac{1}{2} \rho U_{\infty}^{2}\right)=-2\left[f+(1-\xi) f^{\prime}\right] .
$$

The function $f$ may be evaluated from the data by integrating the experimental values for $f^{\prime}$. Despite the delicacy of this calculation, the results for the second and third stations are in excellent agreement after a suitable adjustment to satisfy the necessary conditions $f(0)=f(\infty)=0$ which are implicit in (4). The associated curve for $C_{p}$ from $(5)$ is plotted in figure 14. The unsteady terms $f$ and $\xi f^{\prime}$ turn out to be appreciable. Under the assumption that $\partial p / \partial y$ can be neglected, this curve is the mean pressure signature that should be observed at the wall in the plane of symmetry of the spot. Incidentally, a reasonable estimate for $\partial p / \partial x$, using (5) and figure 14, supports the conclusion that streamwise pressure gradients within the spot are probably not important for the dynamics.

Some small but definite effects of the Reynolds number appear in the present data. At issue is the accuracy of the assumption of conical similarity (i.e. the assumption of a single length scale $U_{\infty} T$ ). In figures 13 and 14, the location of the knee near the wall in loci $A$ and $B$ apparently varies with the finite thickness of the ambient laminar layer. If so, this knee cannot share the conical property, because its distance from the wall in units of $\eta$ varies like $\xi^{\frac{1}{2}} t^{-\frac{1}{2}}$ and hence vanishes as $t$ becomes large for fixed $\xi$. At the rear, by accident or otherwise, the region of accommodation $F G$ scales with the rest of the spot in conical co-ordinates. A plausible explanation is that relaxation to the normal laminar state occurs in a time proportional to $\delta^{2} / \nu$; but $\delta$ varies like $x^{\frac{1}{2}}$, and hence the relaxation time varies like $x$.

Another effect of Reynolds number appears in figure 15, where the present data are 


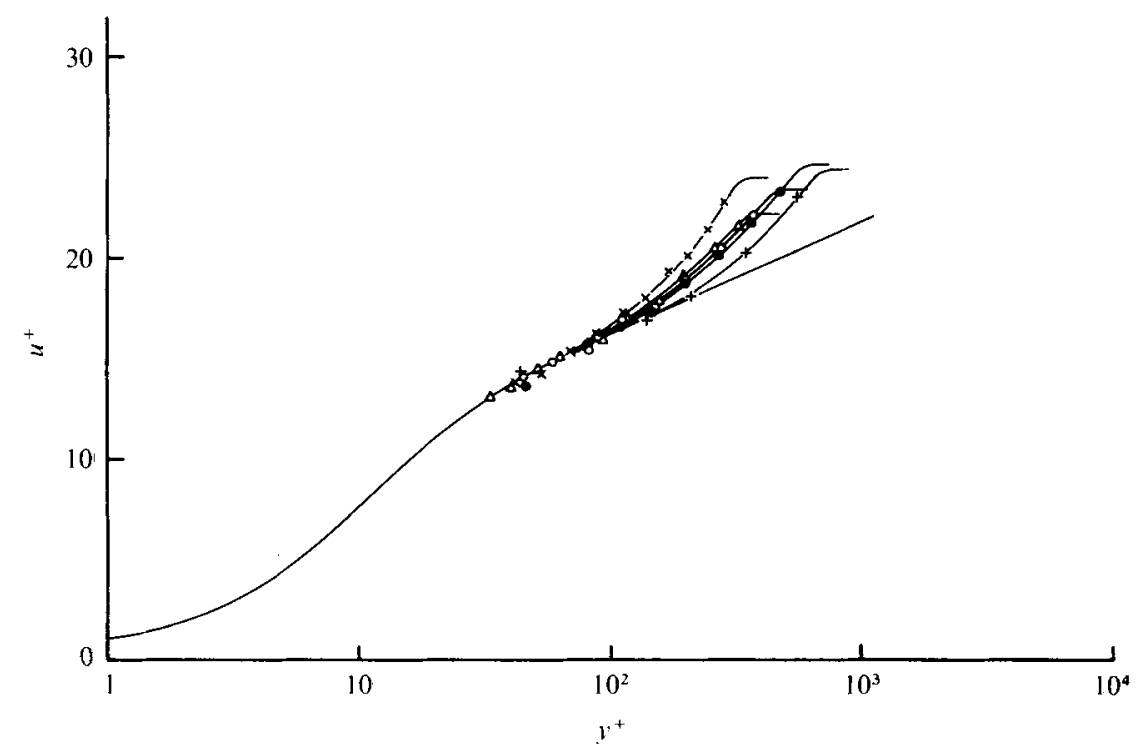

Frgure 15. Mean-velocity profiles along locus $C$ of minimum velocity, plotted in wall variables. Present experiment: $\triangle, x=59 \mathrm{~cm} ; 0, x=89 \mathrm{~cm} ; \bigcirc, x=119 \mathrm{~cm}$. Other experiments: $\times$, Coles \& Barker (1975); + , Wygnanski et al. (1976). Law of the wall is from Coles (1962); in $\log$ region, $u^{+}=(1 / 0 \cdot 41) \ln y^{+}+5 \cdot 0$.

examined (following WSF) through a fit to the law of the wall. The variables are $u^{+}=u / u_{\tau}$ and $y^{+}=u_{\tau} y / \nu$, where $u_{\tau}$ is a parameter which need not necessarily be identified with $\left(\tau_{w} / \rho\right)^{\frac{1}{2}}$. For convenience, the figure shows the velocity variation along the locus $C$ of minimum velocity in figure 14 rather than along a line normal to the wall. Except for possible effects of the pressure gradient, the message of figure 15 is that the present data are not compatible with a momentum-defect law. Both an overall scale $\delta$ and a viscous scale $\nu / u_{\tau}$ are required in order to describe the mean velocity field inside the spot. The data of CB (their figure 2) and the data of WSF (their figure 16) are also included in figure 15. The conclusion by WSF that there is no wake component in the spot seems to be inconsistent with their own data as well as with the present measurements.

\section{The unsteady stream function: similarity}

We now assume the mean flow in the plane of symmetry to be two-dimensional $\dagger$ (cf. figures 23 and $24 b$ of WSF) and introduce a stream function in the customary way by defining

$$
u=\partial \psi / \partial y, \quad v=-\partial \psi / \partial x
$$

Because the flow is not steady, the stream function $\psi$ has the total differential

$$
d \psi=-v d x+u d y+\partial \psi / \partial t d t
$$

$\dagger$ There is persuasive evidence in some of our motion pictures that a version of the turbulent spot which is literally two-dimensional in the mean might be produced by starting with a line disturbance rather than with a point disturbance. The line disturbance might also be swept in $z$ and $t$ to produce a line-vortex structure which is not normal to the direction of the external flow, e.g. a structure with the characteristic angle shown in figure 4(b) (plate 1). 

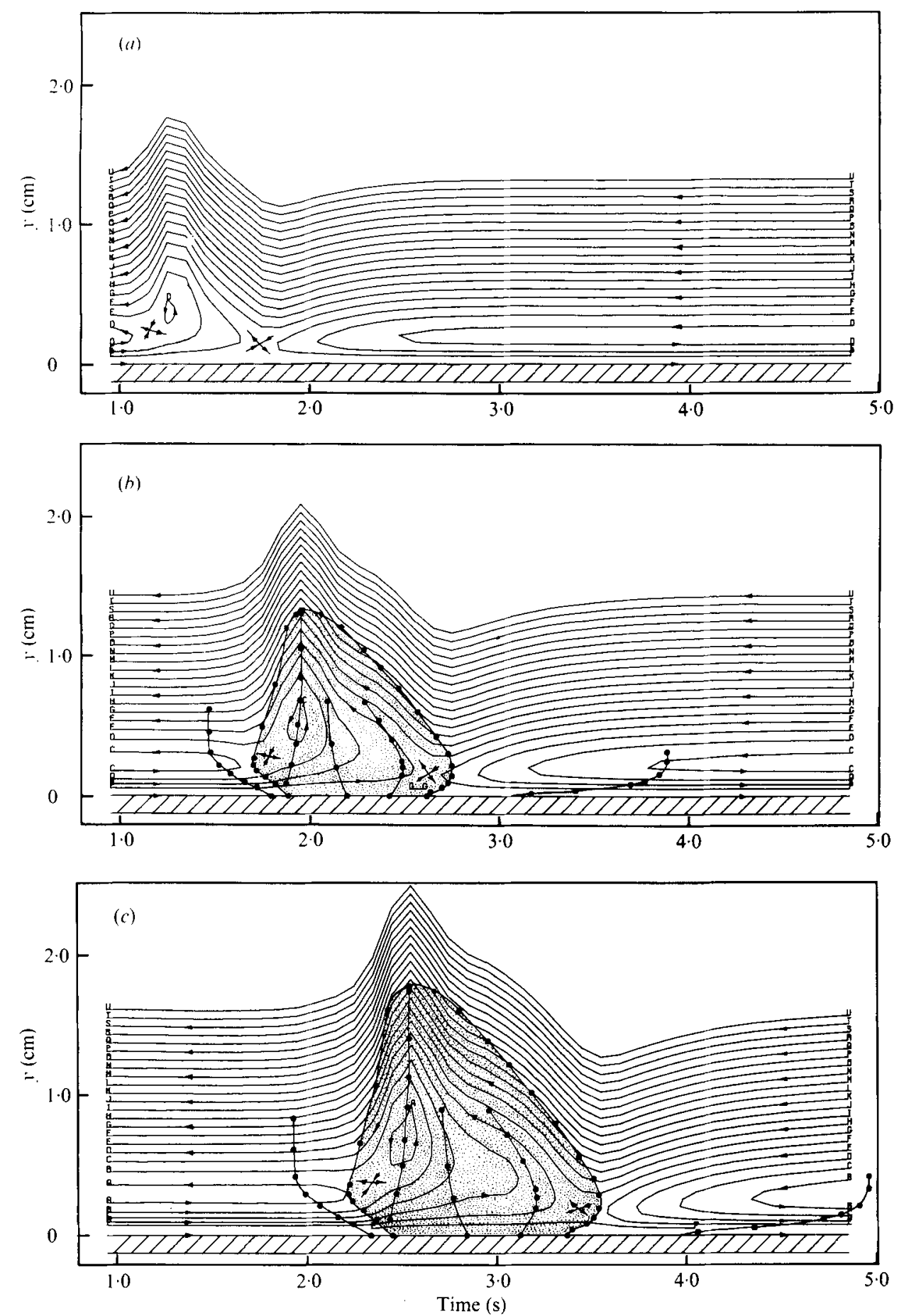

Figure 16. Contours of constant $\psi / U_{\infty}$ in co-ordinates $(y, t)$ as computed for reference frame moving to right at $0.78 U_{\infty}$. (a) $x=59 \mathrm{~cm}$. (b) $x=89 \mathrm{~cm}$. (c) $x=119 \mathrm{~cm}$. 

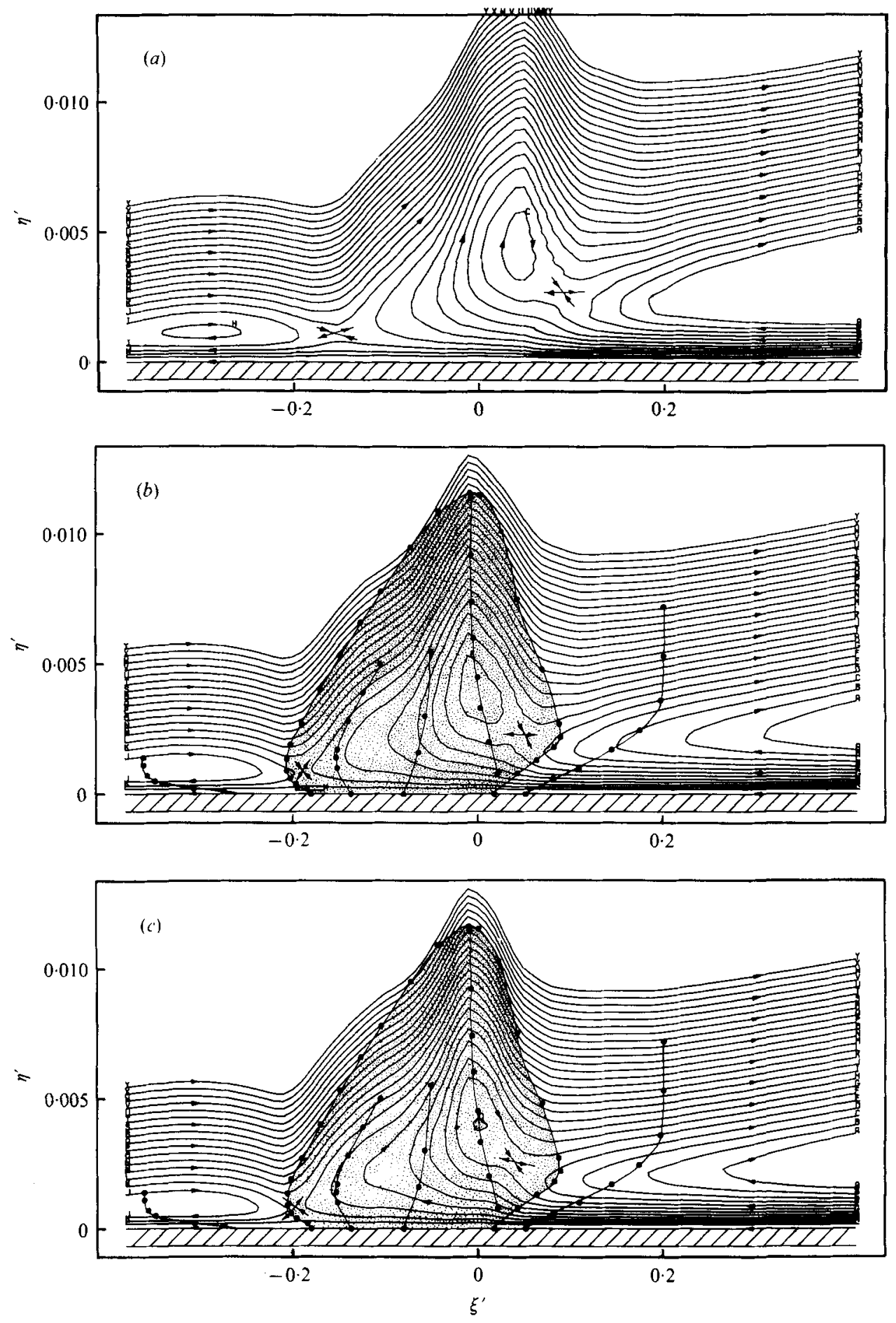

FIGURE 17. Contours of constant $\psi / U_{\infty}^{2} T$ in conical co-ordinates $\left(X / U_{\infty} T, Y / U_{\infty} T\right)$, showing instantaneous streamlines in reference frame moving to right at $0.78 U_{\infty}$. (a) $X=74 \mathrm{~cm}$. (b) $X=104 \mathrm{~cm}$. (c) $X=134 \mathrm{~cm}$. 


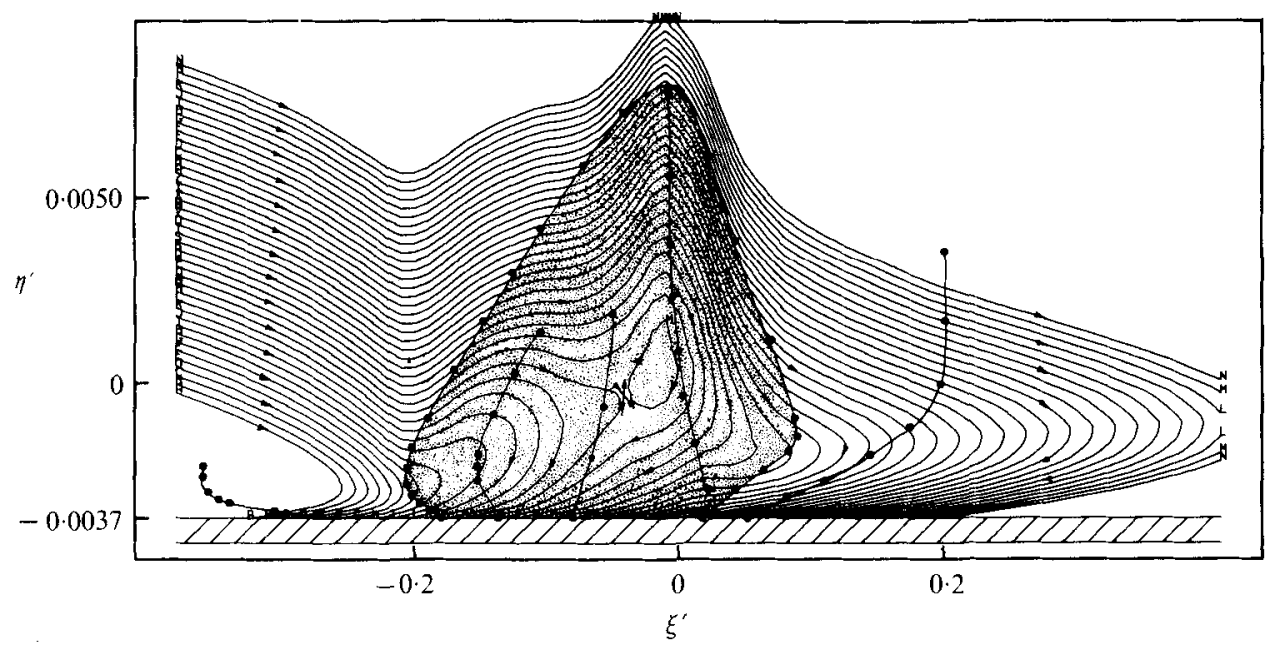

FIGURE 18. Instantaneous streamlines as in figure $17(c)$ but computed for reference frame moving

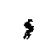
to right at $0.78 U_{\infty}$ and also moving upwards at $0.0037 U_{\infty}$.

Each of the three traverses in figure 11 was made at a fixed value of $x$. If the time $t$ is also held fixed, the stream function $\psi$ can be evaluated from (7) by integration with respect to $y$ of the appropriate instantaneous profile of the mean streamwise velocity $u$. Repetition of this operation for each time step for the data in figure 11 provides a table of values for $\psi$ on a rectangular grid in $y$ and $t$. There are three such tables, one for each measuring station $(x=59,89,119 \mathrm{~cm})$.

Given such an experimentally defined stream function $\psi(x, y, t)$ in laboratory co-ordinates, consider first a Galilean transformation of this stream function to a co-ordinate system which is moving uniformly with positive horizontal and vertical components $c_{1} U_{\infty}$ and $c_{2} U_{\infty}$ respectively (see figure 1 ). Quantities in the moving system (denoted by primes) are related to quantities in the laboratory system by

$$
x^{\prime}=x-c_{1} U_{\infty} t, \quad y^{\prime}=y-c_{2} U_{\infty} t, \quad t^{\prime}=t, \quad \psi^{\prime}=\psi-c_{1} U_{\infty} y+c_{2} U_{\infty} x .
$$

We now assume that the particular values $c_{1}=0.78$ and $c_{2}=0$ select a co-ordinate system which is moving to the right with a velocity characteristic of the large transverse vortex of the spot (cf. the celerity $0 \cdot 77-0 \cdot 80$ associated with locus $C$ in figure 14). When the Galilean transformation is carried out with these values for $c_{1}$ and $c_{2}$, the resulting contours of constant $\psi^{\prime}$ for the three measuring stations are as shown in figure 16. The procedure used to obtain figure 16 makes no use of the conical property and is essentially identical to the procedure used by $C B$ and by $W S F$. The appearance of closed contours suggests the presence of a vortex. However, these contours are not streamlines. Moreover, as pointed out by WSF, the nature of the stream-function contours is critically dependent on the value chosen for $c_{1}$ (and also for $c_{2}$ ). In laboratory co-ordinates, for example, where $c_{1}=c_{2}=0$, no closed contours would appear at all.

Constant values of the stream function $\psi$ define a family of stream surfaces in $x, y, t$ space. Figure 16 amounts to a series of cuts through this family at three successive fixed values of $x$. What is wanted instead is the equivalent of a photographic record of instantaneous streamlines, i.e. a cut through the family of stream surfaces at fixed 
time. The most direct means to this end is use of a similarity transformation based on the property of conical growth which was established in $\$ 5$. We know of only one precedent in the literature of turbulent shear flow for the kind of analysis which occupies the remainder of the present paper. This is Turner's (1964) paper on the turbulent thermal, in which an equivalent transformation is applied, not to real data, but to a hypothetical rising and expanding Hill's spherical vortex.

In the present problem the independent variables are the conical variables $\xi=X / U_{\infty} T$ and $\eta=Y / U_{\infty} T$, where $X=x-x_{0}$ and $T=t-t_{0}$. The appropriate similarity form for the unsteady stream function is

$$
\psi / U_{\infty}^{2} T=g(\xi, \eta)
$$

It is necessary only to convert the tabulated experimental data at any one station to the form $g(\xi, \eta)$ and to find contours of constant $g$ by interpolation. Measurements for fixed $x$ and variable $t$ may now be interpreted as measurements for fixed $t$ and variable $x$, thus allowing the $v$ component of the mean velocity to be calculated from (6). The converted data may be referred to moving (primed) co-ordinates by rewriting (8) in similarity form:

$$
\xi^{\prime}=\xi-c_{1}, \quad \eta^{\prime}=\eta-c_{2}, \quad g^{\prime}=g-c_{1} \eta+c_{2} \xi .
$$

Figure 17 shows contours of constant $g^{\prime}$ obtained by this method for the three measuring stations. The same constant values of $g^{\prime}$ were used to produce each figure, and the values $c_{1}=0 \cdot 78$ and $c_{2}=0$ are the same as those used for figure 16 . For a fixed time, the contours of constant $g^{\prime}$ are instantaneous streamlines as they would appear to an observer in the moving co-ordinate system. If the conical similarity were exact, figures $17(a)-(c)$ would be identical. In reality, the results at the first measuring station are probably characteristic of the initial period of spot development, a period marked by a low speed and a high rate of growth. The results at the last two stations show better agreement. The important point is that the streamline pattern depends on the frame of reference, but the similarity between patterns does not. Nevertheless, figure 17 cannot be used to argue the question of entrainment. Although a contour of constant $g^{\prime}$ does not change position with time, this contour corresponds to different constant values of $\psi^{\prime}$ at different times. The unsteady term in (7) is not accounted for.

In figures $17(b)$ and $(c)$, the closed streamlines are centred at some distance above the plate surface, at roughly $\eta^{\prime}=\eta=0.0037$. If this vertical speed is also taken into account in choosing a moving reference frame (i.e. if $c_{1}=0.78$ and $c_{2}=0.0037$ ), the streamlines for the third measuring station are as shown in figure 18. Relative to this reference frame, the plate appears to be moving to the left and downwards, so that streamlines intersect the plate at a finite angle. The difference between figures $17(c)$ and 18 illustrates the fact that small changes in the speed of the moving frame, particularly in the vertical direction, can cause large changes in the observed streamline pattern. In terms of similarity variables, an equivalent statement is that $g$ is not invariant under translations in $\xi$ and $\eta$.

\section{Particle paths}

The unsteady stream function just defined also contains valuable information about mean particle trajectories. It is clear from figure 9 that fluctuation levels within the spot are high. Turbulent dispersion may therefore cause the mean of an ensemble of 

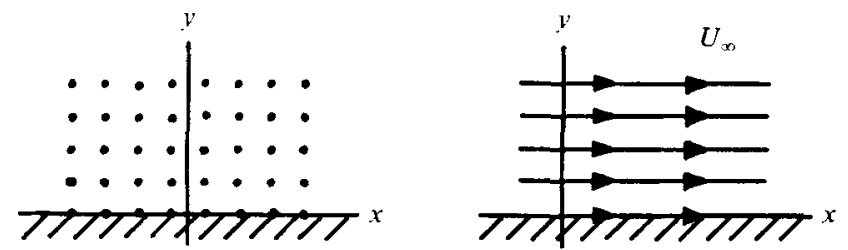

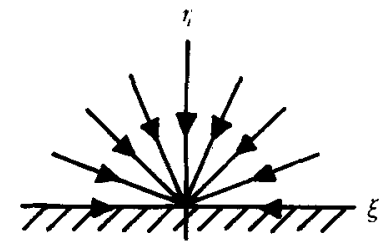

(a)

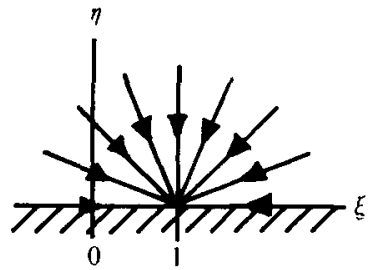

(b)

FIgURE 19. Particle paths in $x, y$ and $\xi, \eta$ co-ordinates for (a) fluid at rest and $(b)$ uniform flow.

particle paths through a given initial point to be quite different from a particle path which is inferred from the mean-velocity field (Hunt \& Mulhearn 1973). For the limited purpose of identifying the main structural properties of the spot, however, we propose to treat the unsteady mean motion as if dispersion were unimportant.

Particle paths in an unsteady two-dimensional flow are defined by the simultaneous equations

$$
\left.\begin{array}{l}
d x(t) / d t=u[x(t), y(t), t], \\
d y(t) / d t=v[x(t), y(t), t] .
\end{array}\right\}
$$

Equations (11) constitute a nonlinear initial-value problem which might be difficult to solve even if $u$ and $v$ were known analytically. In the present situation, $u$ is a measured quantity ( $v$ is inferred from continuity) and is known only at discrete points in the flow. In any event, since the direction of particle paths through a fixed point depends on time, integrated particle paths obtained from (11) would probably contain very little useful information (Hama 1962). Indeed, if the number of particles chosen for the integration were large, the result in $x, y$ co-ordinates would be a tangle of intersecting lines and orbits. Furthermore, the pattern of particle paths, like the pattern of streamlines, would depend on the speed of a moving observer.

Most of these difficulties disappear when the conical-similarity form (9) is used for the stream function. Similarity is a powerful organizing influence in any experimental situation, and the stream function itself guarantees continuity. Let (11) be rewritten in terms of $\xi, \eta$ and $g(\xi, \eta)$ :

$$
\left.\begin{array}{l}
d \xi / d \tau=g_{\eta}-\xi=u / U_{\infty}-\xi \\
d \eta / d \tau=-g_{\xi}-\eta=v / U_{\infty}-\eta
\end{array}\right\}
$$

where $\tau=\ln T$. Equations (12) have the property that the right-hand sides do not depend explicitly on the independent variable $\tau$. Consequently, while the position of a particle in $\xi, \eta$ space varies with time, the trajectory of the particle does not. In fact, not only are particle trajectories in $\xi, \eta$ space independent of time, but they are also independent of the speed of a uniformly moving observer. This property follows directly from 


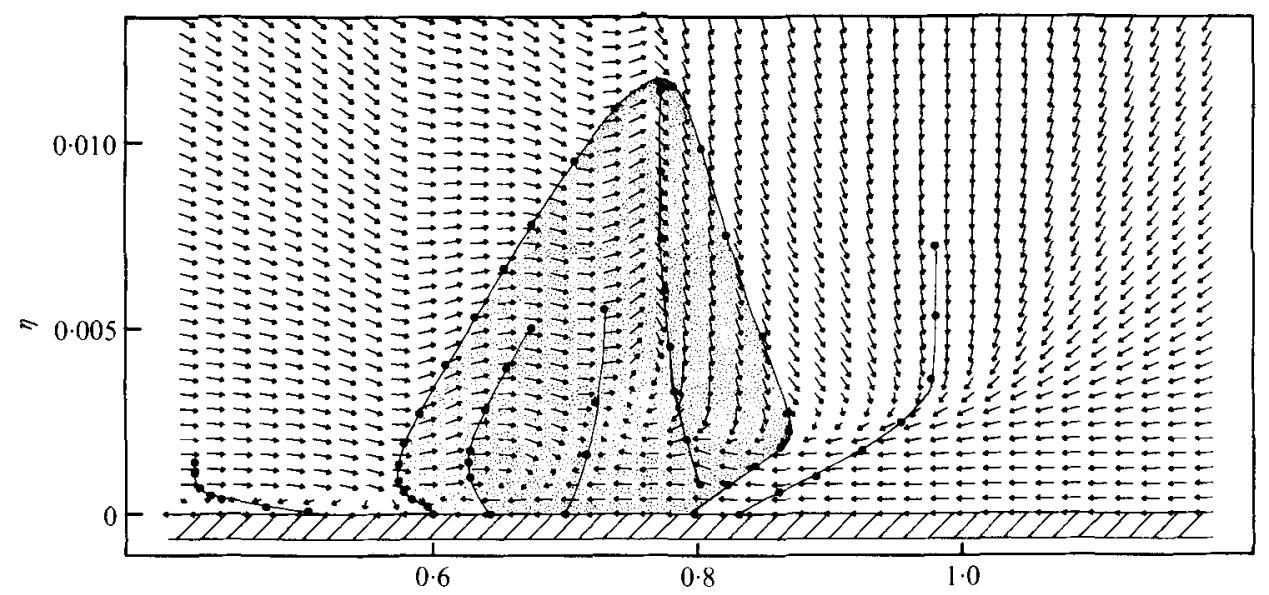

(a)

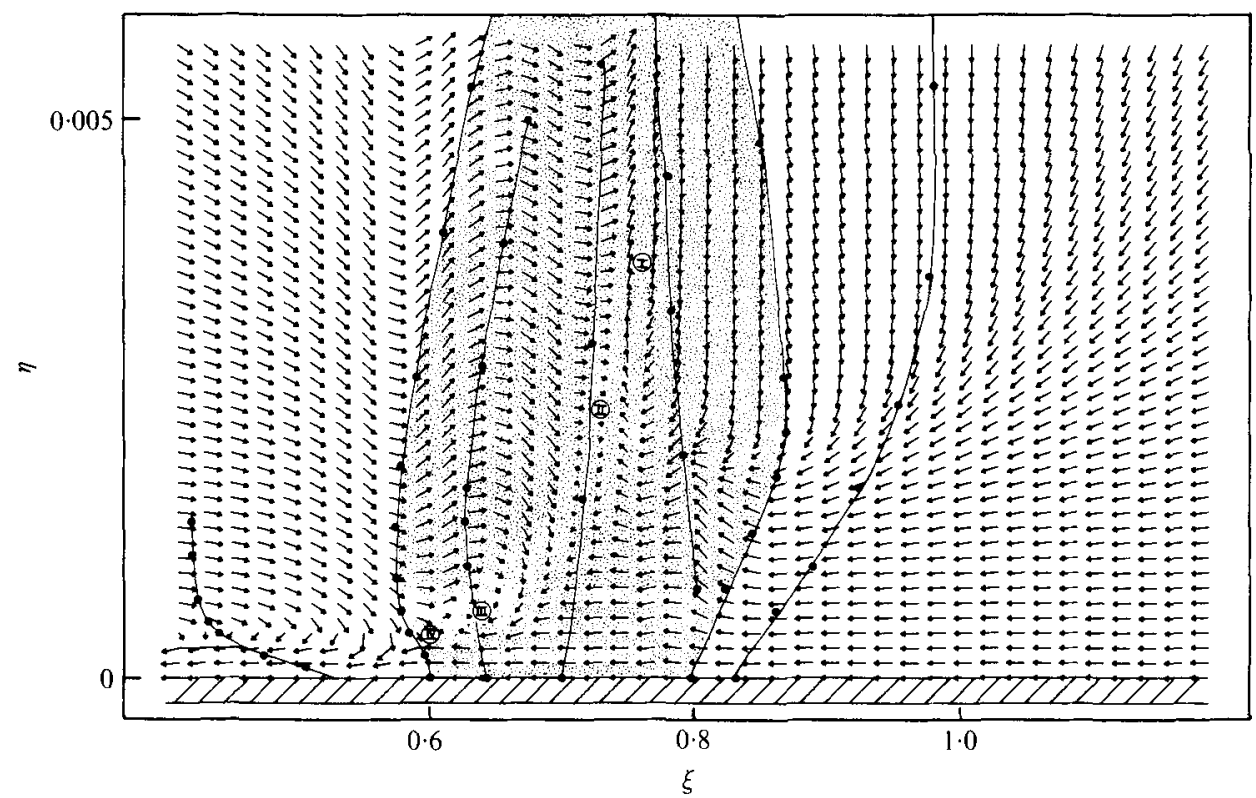

(b)

Frgure 20. Displacement-vector field in conical co-ordinates for the data at $X=134 \mathrm{~cm}$. (a) Every third data point plotted. (b) Ordinate expanded by a factor of three; every data point plotted.

(10), which imply $g_{\eta^{\prime}}^{\prime}-\xi^{\prime}=g_{\eta}-c_{1}-\xi+c_{1}=g_{\eta}-\xi$ and $g_{\xi^{\prime}}^{\prime}+\eta^{\prime}=g_{\xi}+c_{2}+\eta-c_{2}=g_{\xi}+\eta$. All observers, moving or not, would assign the same numerical values to the quantities $-g_{\xi^{\prime}}^{\prime}-\eta^{\prime}$ and $g_{\eta^{\prime}}^{\prime}-\xi^{\prime}$ and to their ratio $d \eta^{\prime} / d \xi^{\prime}=d \eta / d \xi$. A moving observer would assign these values at points which are uniformly displaced by fixed amounts $c_{1}$ in $\xi$ and $c_{2}$ in $\eta$, but this displacement would not affect the pattern of particle trajectories.

In effect, the conical transformation can be viewed as a zoom transformation in which the observer is receding in the $z$ direction, out of the plane of the motion, at a rate which freezes the apparent size of any linearly growing features of the flow.

It may be helpful to illustrate the nature of conical similarity as a zoom transforma- 
tion by considering two simple cases. These are the case of fluid at rest $(\psi=g=0)$ and the case of fluid moving uniformly at speed $U_{\infty}\left(\psi=U_{\infty} y=U_{\infty}^{2} t \eta ; g=\eta\right)$. Equations (12) then become

for the first case and

$$
\mathrm{d} \xi / d \tau=-\xi, \quad d \eta / d \tau=-\eta
$$

for the second.

$$
d \xi / d \tau=1-\xi, \quad d \eta / d \tau=-\eta
$$

Particle paths in $x, y$ and $\xi, \eta$ co-ordinates for the two flows are shown in figure 19. It is clear that a receding observer need not make a distinction between a uniform flow and no flow at all; the pattern of particle paths is the same in both cases. Consequently, there is no loss of generality in supposing that the observer is at rest in $x, y$ co-ordinates. We emphasize that the flow in figure 19 is not a sink flow and that the velocity can not be inferred from the separation between adjacent particle paths as if the flow were steady.

Finally, note that an isolated turbulent spot can be thought of, in either the analytical or the experimental context, as a moving perturbation to a uniform flow. Far from the point $(1,0)$ in $\xi, \eta$ space, therefore, particle paths can be expected to behave asymptotically like the rays in figure $19(b)$, whatever the details of the flow in the spot (cf. figures 20 and 21).

\section{Structure}

In the present instance, it is convenient to solve the particle-path equations (12) graphically by the method of isoclines. The method is illustrated in figure $20(a)$ for the data at the third measuring station. Figure $20(b)$ is similar except that the ordinate has been expanded by a factor of three to show more detail near the wall. Each grid point in $\xi, \eta$ co-ordinates has affixed to it the tail of an arrow whose head points in the direction of increasing time. The slope of a given arrow is $d \eta / d \xi=\left(-g_{\xi}-\eta\right) /\left(g_{\eta}-\xi\right)$ and its length is proportional to the local rate of displacement. Because the relative displacements cover a wide range of magnitudes, a cut-off length is used to avoid overlapping arrows. However, relative displacements are correctly shown near critical points, i.e. points where $d \eta / d \xi$ has the form $0 / 0$. Far from the critical points, the actual rates of displacement may be several times larger than those shown.

The critical points were located as intersections of boundaries separating positive and negative values of the two functions $g_{\eta}-\xi$ and $-g_{\xi}-\eta$. Slopes in the neighbourhood of the intersections were then used to classify these points (see appendix). The spot turns out to contain four critical points. There are two saddles, or stagnation points, and two stable foci, $\dagger$ or points of accumulation. These are labelled I, II, III and IV in figure $20(b)$.

Representative particle paths are sketched in figure 21 with the critical points placed according to the data at the third measuring station. This figure is the main result of the present paper. The particle paths are shown as dashed lines inside the body of the spot to indicate that turbulent dispersion has been neglected.

$\uparrow$ The term stable focus may seem to be unnecessarily formal. However, in the mathematics of autonomous systems, the term vortex point is sometimes used to describe a critical point which is at the centre of a family of closed orbits. To avoid confusion, we take the more formal approach here. 


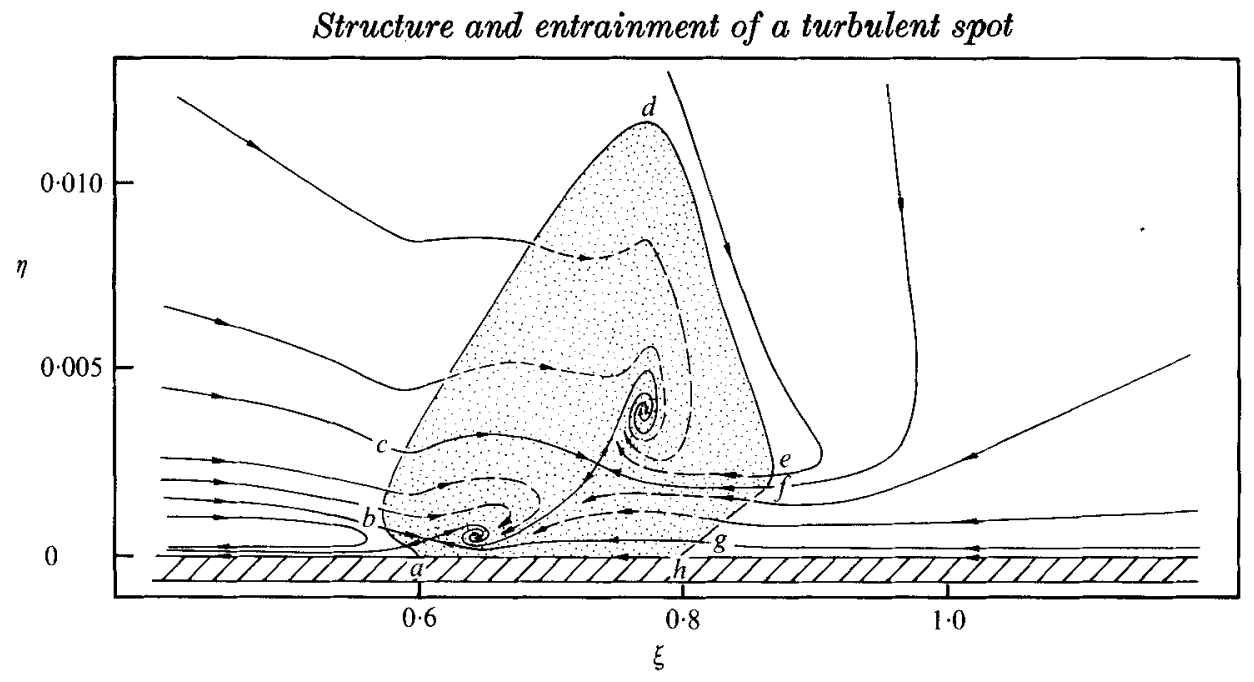

663

FIGURE 21. Sketch of particle trajectories, with critical points located and classified from the data.

Several useful conclusions with respect to structure follow from the analysis so far:

(i) Comparison of figure 21 with figure 17 (or figure 18) confirms that lines of constant $g$ are not particle paths, nor are they tangential to particle paths. The reason is explicit in (12); the rate of displacement of a particle in $\xi, \eta$ space is the vector sum of the velocity field defined by $g$ (with components $g_{\eta},-g_{\xi}$ ) and the apparent velocity field induced by the recession velocity of the observer (with components $-\xi,-\eta$ ).

(ii) There are actually two vortex structures associated with the average spot. One is the large transverse vortex (focus $I$ in figure $20 b$ ) which was previously identified by CB. The other, close to the wall near the rear of the spot, is a secondary vortex (focus III in figure 20b) which probably influenced the measurements of celerity by WSF.

(iii) The foci in figure 21 do not violate continuity, nor do they imply three-dimensional mean flow. They are points of accumulation as they would appear to an observer who is receding out of the plane of the flow in a way which preserves the linear dimensions of the spot. Figure $19(b)$, which shows particle paths for uniform flow, where the fluid appears to vanish into a sink at $(1,0)$, underlines this point.

(iv) Figure 21 is also a generalized celerity plot for the turbulent spot. Each point $(\xi, \eta)$ defines its own celerity. In particular, the primary focus I moves with celerity $c_{1}=0.77, c_{2}=0.0038$ (cf. the original estimate $c_{1}=0.78, c_{2}=0.0037$ in $\S 6$ ), while the secondary focus III moves with celerity $c_{1}=0.64, c_{2}=0.0006$. These celerities do not depend on the speed of a uniformly moving observer.

These conclusions are possible only because the structure of the spot is remarkably stable and permanent. This structure is not noticeably strained or distorted by either internal or external mean motion. The ambient flow accommodates to the presence of the spot by submitting to entrainment or by passing over or under the spot vortices.

\section{Entrainment}

Entrainment and growth are characteristic of all unconfined Qurbulent shear flows. Entrainment rates have traditionally been defined in terms of a volume or mass balance based solely on properties of the mean flow. While this definition is quite 


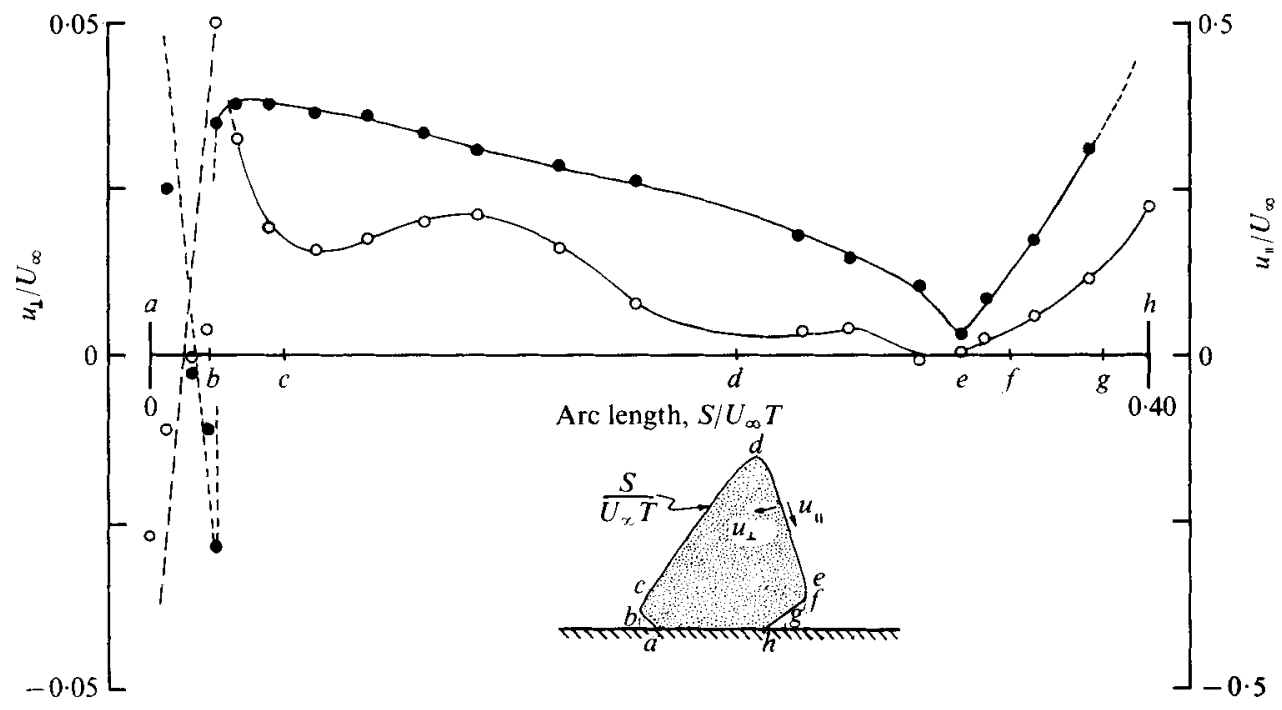

Figure 22. Normal and tangential velocity components of fluid relative to spot boundary. $\bigcirc, u_{\perp} / U_{\infty} ; \mathbf{O}, u_{\|} / U_{\infty}$.

useful for engineering purposes, it says nothing about the actual physical processes which govern entrainment. Since the work of Corrsin \& Kistler (1954), entrainment has also come to mean propagation of a thin laminar-turbulent interface into nonturbulent fluid by viscous diffusion of vorticity, a process sometimes called nibbling. According to this view, entrainment rates are controlled by the properties of smallscale turbulence in the neighbourhood of the interface. In the mean and on a large scale the interface can be thought of as essentially flat. It entrains over a relatively large area by propagating normal to itself relatively slowly compared with the absolute mean velocities in the same flow. Hence fluid often tends to cross the mean interface at a quite shallow angle.

A somewhat different view has been proposed by some proponents of large-eddy models of turbulence (e.g. Bevilaqua \& Lykoudis 1971; Brown \& Roshko 1974). The assertion here is that entrainment usually also involves a large-scale process sometimes called engulfment, or gulping, in which non-turbulent fluid is incorporated into the turbulence by a folding process analogous to the rolling-up of a rug. According to this view, entrainment is directly controlled by the motion induced by large vortex structures in the flow. Non-turbulent fluid is considered to be committed to eventual entrainment well before it is actually entrained in the sense of acquiring threedimensional vorticity at small scales. This view, like the previous one, is an oversimplification of a complicated phenomenon, and the two views may in fact differ mainly in the scale of the process being described.

Having figure 21 in hand, we are in a position to make quantitative statements about entrainment for the turbulent spot, at least in the plane of symmetry. It should be kept in mind that the spot boundary is defined here in terms of features of the meanvelocity field (see $\$ 5$ ) and may not coincide with the mean position of the laminarturbulent interface, however the latter might be defined. With this reservation, the $x, y$ components of the dimensionless velocity of the spot boundary itself are simply the $\xi, \eta$ co-ordinates at any point. Within the fluid, the $x, y$ components of velocity relative 


\begin{tabular}{|c|c|c|c|c|c|}
\hline Region & Arc & $\frac{A}{U_{\infty}^{2} T^{2}}$ & $\frac{1}{U_{\infty}^{2} T} \frac{d A}{d T}$ & $\int \frac{u_{\perp}}{U_{\infty}} d\left(\frac{S}{U_{\infty} T}\right)$ & Destination \\
\hline Outer & $\begin{array}{l}c d \\
d e \\
e f\end{array}$ & 0.00146 & 0.0029 & $\left.\begin{array}{l}0.0025 \\
0.0002 \\
0.0001\end{array}\right\} 0.0028$ & Focus I \\
\hline Intermediate & $\begin{array}{l}b c \\
f g\end{array}$ & 0.00054 & $0 \cdot 0011$ & $\left.\begin{array}{l}0.0010 \\
0.0003\end{array}\right\}^{0.0013}$ & Focus III \\
\hline Wall & $\begin{array}{l}a b \\
g h\end{array}$ & 0.00007 & $0 \cdot 0001$ & $\left.\begin{array}{r}-0.0001 \\
0.0003\end{array}\right\}^{0.0002}$ & Through flow \\
\hline Total & $a h$ & 0.00207 & $\begin{array}{r}0.0041 \\
\text { TabIE } 1\end{array}$ & 0.0043 & \\
\hline
\end{tabular}

to the boundary are given by (12) as $g_{\eta}-\xi$ and $-g_{\xi}-\eta$. It remains only to calculate the components of relative velocity for axes normal and parallel to the boundary at each point. The results are plotted in figure 22 as a function of are length, beginning at the rear of the spot.

Integration in figure 22 leads to the estimated entrainment rates listed in table 1, where the symbol $A$ refers to the area of the particular region in question and $S$ is the arc length along the spot boundary. In figure 21 the spot is divided into outer, intermediate and wall regions by the two horizontally running separatrices which pass through the saddles II and IV originally defined in figure $20(b)$. The rates for the three regions are listed separately in the table. There is good overall agreement between the growth rate computed by integrating local entrainment rates and the growth rate required by the property of conical similarity. Since these two rates are determined almost independently, the agreement supports the assumptions of the analysis. It is possible that the wall region, and perhaps the intermediate region as well, will make up a smaller and smaller fraction of the spot as time and the Reynolds number increase. In any event, the results presented in figures 21 and 22 and in table 1 are submitted as a valid description of structure and entrainment for the limited time of observation represented by the present data.

Figure 22 and table 1 show that entrainment is far from uniform around the periphery of the spot. In particular, our data do not confirm a conclusion reached by WSF, that there is appreciable entrainment along the outer front boundary (the segment $d e$ ). More than $80 \%$ of the entrainment occurs by the process called nibbling along the upper rear boundary (the segment $b c d$ ). The remainder occurs near the wall at the front of the spot (the segment efg), but not necessarily by the process called gulping. It seems to be primarily viscous friction at the wall which brings this fluid within range of the spot, rather than motion induced by the large-eddy structure.

\section{Discussion}

The original objective of the present research was to study entrainment by exploiting the conical property of spot growth, in order to advance to the concept of instantaneous mean streamlines and to the more powerful concept of mean particle trajectories. As a result of the research, other prospects and other problems have appeared. For example, a knowledge of particle trajectories carries with it the prospect of eventual 
access to dynamies, in terms of the description by the unsteady Reynolds equations of dynamical processes which act on a fluid element as it moves along a mean particle trajectory. The turbulent spot is a coherent structure which is filled with fluctuations. These fluctuations ought to be describable in the usual way, in terms of turbulent energy, turbulent shearing stress and turbulent dissipation. However, the concept of Reynolds stress now appears at a structural level one step below the conventional level. Fluctuations are no longer to be measured with respect to a conventional long-time average. Instead, they are to be measured with respect to an average over an ensemble of realizations, with phase information retained at the largest scale.

Unfortunately, the information available from the present measurements is essentially kinematic information. Neither our experiment nor any other experiment at present provides the information required for a competent discussion of dynamics. What is missing is information about velocity-velocity covariances and perhaps velocity-vorticity covariances of fluctuations at the lower structural level just defined. It would also be useful to know the distribution of wall shearing stress and to have more information about the pressure field.

A few qualitative inferences can be drawn about dynamics by combining the kinematic evidence from $\S \S 8$ and 9 with the visual evidence from $\S 3$. The discussion is imprecise for at least three reasons. First, the mean flow in the plane of symmetry is unlikely to be accurately two-dimensional. Second, the assumption of conical similarity is demonstrably not exact. Third, ensemble-mean particle paths may differ substantially from particle paths calculated from the ensemble-mean velocity field. One positive element in the discussion is the real scale of the cross-section of the spot in undistorted co-ordinates, as illustrated by the photograph in figure 7(a) (plate 4) and by the sketch at the top of figure 14. This cross-section is sufficiently flat that it may be both useful and permissible to think in terms of a boundary-layer approximation involving nearly rectilinear flow in laboratory co-ordinates.

Consider the processes which act on free-stream fluid entering the spot along the rear interface $b c d$ in figure 21 . Whether this fluid on the average winds up in focus $I$, with a final velocity $0 \cdot 77$, or in focus III, with a final velocity $0 \cdot 64$, may not be important. What is important is that this fluid undergoes a violent introduction to turbulence as it is entrained at the boundary $F$. The fluid loses momentum rapidly between $F$ and $E$ (see the appropriate parts of traces $\gamma$ and $\delta$ in figure 12). Given the boundary-layer approximation, it follows that much of the lost momentum must be transferred to fluid closer to the wall. It is this transfer which accounts for the high shearing stress near the tail of the spot, in the vicinity of focus III. A high stress level implies high turbulence production and active turbulence (at intermediate and small scales) which is able to propagate into the non-turbulent fluid at a speed sufficient to hold the rear boundary $F$ fixed in similarity co-ordinates. According to figure 22, the propagation velocity of the interface $F$ normal to itself is about $0.02 U_{\infty}$, i.e. about half of $u_{\tau}$ (cf. $U_{\infty} / u_{\tau} \sim 25$ in figure 15). This description also suggests a mechanism to account for the finite speed of the spot at the rear. If the tail were to lag relative to its normal position, the slower gradients in the stream direction inside the spot would signify a reduced rate of momentum loss and thus a reduced stress level. With or without a decrease in $\partial u / \partial y$, the turbulence production and turbulence intensity would decrease, and the rear interface would propagate more slowly, restoring the status quo. It is also worth noting that fluid entrained at the rear of the spot overtakes the main vortex relatively 
slowly. Estimates of the associated transit time might be useful in studies of relaxation rates for large-eddy structures in more general flows.

At the front of the spot, there is room for argument as to whether or not the outer part of the front accommodation region $A B$ should be considered to be outside the body of the spot. Our view is that turbulence in this region must be capable of transport, because there is a weak momentum loss for part $A B$ of traces $\gamma$ and $\delta$ in figure 12 . However, the nature of the particle trajectories in figure 21 suggests that this turbulence must be decaying, at least in the outer part of the flow, with energy only at low frequencies (of. figure $7 a$, plate 4 ).

Entrainment also occurs as the spot overruns slower vorticity-bearing fluid in the ambient laminar boundary layer. In the mean, according to figure 21, this entrained fluid can be divided into three layers. The two upper layers are swept up and incorporated into the spot, while the lowest layer emerges intact (in the mean sense) at the rear. In reality, however, the aluminium flow visualization shows the motion in this lowest layer to be quite complicated, and some further discussion is appropriate.

One of us (DC) has proposed on several public occasions, beginning with a Rand workshop on low-speed boundary-layer transition in 1974, that sublayer streaks may be longitudinal vortices caused by an instability of Taylor-Görtler type. This conjecture has also recently been advanced independently by Brown \& Thomas (1977). To see the force of the conjecture, consider the kinematics of the mean flow very near the wall during the passage of a turbulent spot. Suppose that a fluid element in the laminar layer just ahead of the spot is initially moving at a velocity small compared with $U_{\infty}$. When it is overtaken by the spot, the element must accelerate rapidly. This acceleration occurs between loci $A$ and $B$ in figure 14 and is evident in trace $\alpha$ in figure 12. Because of continuity, the element must also move much closer to the wall. Now it is a necessary condition for Taylor-Görtler instability that particle trajectories have finite curvature (necessarily concave to the free stream in the present case). In the two-dimensional mean flow in and near the sublayer, the trajectories during the latter part of the motion just described have curvature of the proper sign to allow the instability. Evidence that an instability actually occurs is given by the presence of streamwise precursor vortices under the overhanging nose of the spot in figures 6 and 7 (plates 3 and 4). On the other hand, the conjecture so far does not include a mechanism to maintain the curvature of the mean trajectories, at least locally, and thus keep the sublayer vortices active under the spot. Without such a mechanism, only visual evidence is relevant.

A remarkable property of the sublayer structure in figures 6 and 7 is the high degree of homogeneity in space, $\uparrow$ without any conspicuous indication that the unseen main flow contains motions on a much larger scale. This property is especially surprising in the case of the contamination wedge (figure 6 , plate 3 ), which other evidence suggests may be made up of isolated spot-like structures (of. figure $4 b$, plate 1). Part of the explanation may be that the streaks or vortices are sometimes being energized and sometimes not. A careful study of the aluminium photographs is required to conclude that active and passive regions can in fact be distinguished, but not well enough to infer the presence in certain regions of larger structures further from the wall.

$\dagger$ This property of homogeneity is implicit in the work by the Stanford group. For example, Kline et al. (1967) were apparently able to count sublayer dye streaks in motion-picture frames selected at random. 
At the rear boundary of the spot, fluid being left behind close to the wall must eventually reconstitute part of the original laminar layer. To the extent that it has participated in the turbulent motion, this fluid is full of fluctuations. If these are primarily due to longitudinal vortices in the sublayer, it is reasonable to find that weak and fairly regular streamwise secondary vorticity is present in the rear accommodation region $F G$. The flow visualization in figures 6 and 7 does show such vortices. As this secondary vorticity decays, it also supports a Reynolds stress by transporting fluid towards and away from the wall. This transport explains how the flow in the wake or calming region might possess an abnormally full profile without the benefit of a pressure gradient. The characteristic time for decay of these vortices is selfevident in figure 11. At each station, the decay time is close to the original diffusion time of the laminar boundary layer, which is by definition $x / U_{\infty}$ and thus is also the waiting time before arrival of the spot at the station in question.

One remaining area of uncertainty is the meaning of the second focus III in figure 21 . It is not an obvious feature. In fact, it was overlooked at first, and appeared only when the scale was made finer in going from figure $20(a)$ to figure $20(b)$ in an attempt to account for loci $D$ and $E$ in figure 14. This second focus seems to evolve with increasing Reynolds number and to be best defined at the third station. It is possible that focus III appears as a precursor to splitting (more accurately, mitosis) and that more than one large eddy might appear in a single spot at much higher Reynolds numbers if these were experimentally accessible. It is also possible that focus III is simply the terminal feature of the sublayer flow and is part of whatever mechanism shuts off the input of energy to the sublayer vortices at the position of maximum wall shearing stress.

Is the turbulent spot a prototype large eddy for the turbulent boundary layer? In some respects we believe that it is. There is the feature of a large internal vortex, occupying much of the cross-section. There is also the localization of entrainment over the rear interface. On the other hand, an isolated mature spot is much too large, especially in the spanwise direction, to fit comfortably in a boundary layer. Extrapolation of the mean-velocity profiles in figure 15 to larger Reynolds numbers also suggests that the vortex motion (as represented by the wake component of the profile) can become more vigorous than might be expected for a corresponding large-eddy structure in a boundary layer. Perhaps it might be more useful, especially at large Reynolds numbers, to think of the turbulent spot as simply an alternative flow to the boundary layer.

Finally, we emphasize once more that the essence of this paper is the application of a similarity transformation to experimental data. The basis for the transformation technique is the fact that the nonlinear particle-path equations are reduced by the similarity assumption to an autonomous system. This fact allows the motion to be analysed in the phase plane defined by the two similarity variables and permits the large-eddy structure to be described in terms of the unifying concept of critical points. The whole machinery of the mathematics of autonomous systems, a machinery which has been quite productive in other fields of nonlinear mechanics, might be made available to the field of turbulence at the slight cost of neglecting effects of finite Reynolds number on the large-eddy structure. It is even possible that the transformation technique may eventually be capable of associating growth laws based on global similarity arguments with growth laws based on the idea of local entrainment by coherent structures. We therefore believe that this technique can play an important role in future research on turbulent shear flow. 


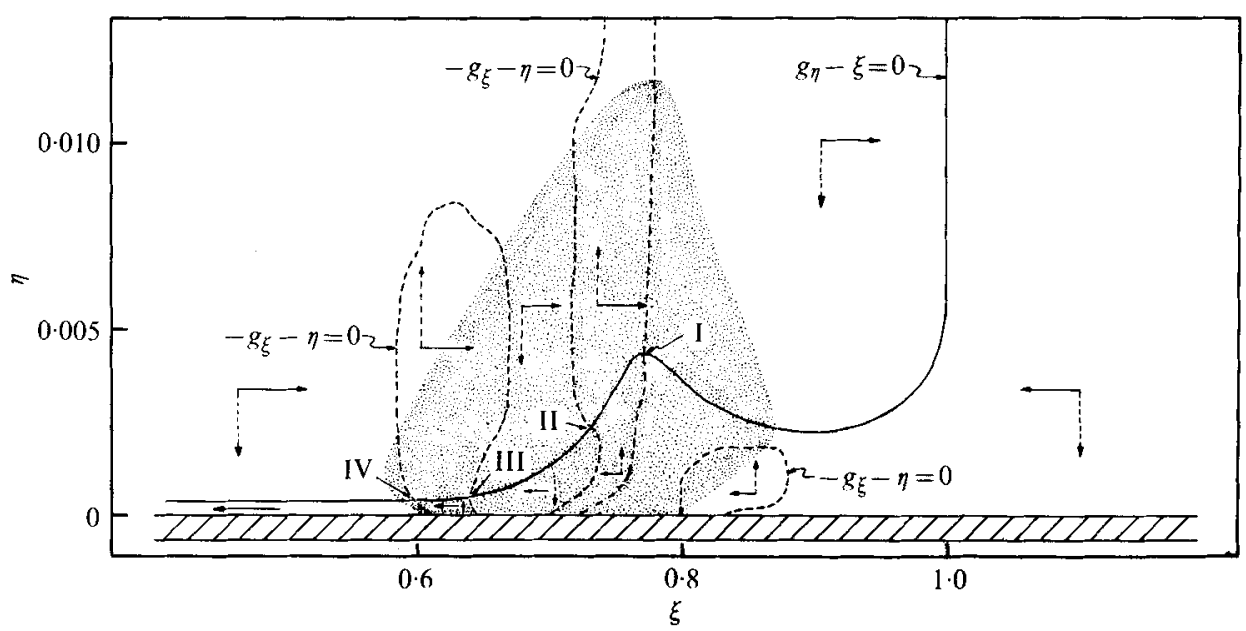

FrguRE 23. Diagram showing how critical points were located. Solid arrows indicate direction of horizontal component of particle displacement. Dashed arrows indicate direction of vertical components of particle displacement.

This research was supported by the National Science Foundation under grant ENG75-03694.

\section{Appendix}

\section{By Brian CaNTwEll}

The basis for the application of phase-plane techniques to the entrainment problem is the fact that particle paths in fluid flows satisfy ordinary differential equations, namely

$$
d x / d t=u(x, y, z, t), \quad d y / d t=v(x, y, z, t), \quad d z / d t=w(x, y, z, t) .
$$

If the flow is steady, then the above equations reduce to an autonomous set with particle trajectories which are identical to streamlines. If the flow is unsteady, then the reduction can be accomplished with the aid of a similarity transformation. It should be noted that the similarity form (9) used to fit the data in this experiment is not simply an ad hoc representation for the stream function, but a valid similarity transformation which can be used to reduce the number of independent variables in the two-dimensional Euler equation by one (Cantwell 1978).

A great deal can be learned about the solutions to the particle-path equations by locating critical points and examining the slopes of the integral curves near these points (Perry \& Fairlie 1974). Figure 23 shows schematically how the critical points were located. The solid line in figure 23 is the line of demarcation between positive and negative data for the quantity $g_{\eta}-\xi$ while the dashed line is the line of demarcation between positive and negative data for the quantity $-g_{\xi}-\eta$. The critical points lie at the intersections of the two lines. In order to classify the points, we assume that $g_{\eta}-\xi$ and $-g_{\xi}-\eta$ are regular in the neighbourhood of a critical point, i.e.

$$
\left[\begin{array}{l}
d \xi / d \tau \\
d \eta / d \tau
\end{array}\right]=\left[\begin{array}{ll}
a & b \\
c & d
\end{array}\right]\left[\begin{array}{c}
\xi-\xi_{0} \\
\eta-\eta_{0}
\end{array}\right]=\mathbf{A}\left[\begin{array}{c}
\xi-\xi_{0} \\
\eta-\eta_{0}
\end{array}\right]
$$

where $\xi_{0}$ and $\eta_{0}$ are the co-ordinates of the point. 


\begin{tabular}{|c|c|c|c|c|c|c|c|c|c|c|c|c|c|}
\hline & \multicolumn{2}{|c|}{$\overbrace{}^{\xi_{0}}$} & \multicolumn{2}{|c|}{$\eta_{0}$} & \multicolumn{2}{|c|}{$a$} & \multicolumn{2}{|c|}{$b$} & \multicolumn{2}{|c|}{$c$} & \multicolumn{2}{|c|}{$d$} & \multirow[b]{2}{*}{ Type } \\
\hline & 89 & 119 & 89 & 119 & 89 & 119 & 89 & 119 & 89 & 119 & 89 & 119 & \\
\hline I & 0.77 & 0.77 & 0.0037 & 0.0038 & $-2 \cdot 2$ & $-2 \cdot 0$ & 56 & 61 & -0.2 & $-0 \cdot 3$ & 0.5 & -0.1 & $\begin{array}{r}\text { Stable } \\
\text { focus }\end{array}$ \\
\hline II & 0.71 & 0.74 & 0.0013 & 0.0024 & $-1 \cdot 7$ & $-2 \cdot 3$ & 91 & 58 & 0.04 & $0 \cdot 04$ & 0.4 & $0 \cdot 3$ & Saddle \\
\hline III & 0.65 & 0.64 & 0.00061 & 0.00061 & $-2 \cdot 3$ & $-1 \cdot 8$ & 264 & 254 & $-0 \cdot 02$ & -0.02 & $0 \cdot 2$ & $-0 \cdot 3$ & $\begin{array}{r}\text { Stable } \\
\text { focus }\end{array}$ \\
\hline IV & 0.62 & $0 \cdot 61$ & $0 \cdot 00047$ & $0 \cdot 00041$ & $-2 \cdot 3$ & $-2 \cdot 7$ & 457 & 467 & 0.01 & 0.01 & $0 \cdot 2$ & 0.7 & Saddle \\
\hline
\end{tabular}

The nature of the critical points can be established from the trace $p=a+d$ and determinant $q=a d-b c$ of the matrix A. The elements of $\mathbf{A}$ for each of the four singularities in the spot have been computed for the data at the last two measuring stations. See table 2.

The velocity, vorticity and rate-of-strain fields in the neighbourhood of the critical point are computed as follows:

$$
\begin{aligned}
& u / u_{\infty}=g_{\eta}=\xi+a\left(\xi-\xi_{0}\right)+b\left(\eta-\eta_{0}\right), \\
& v / u_{\infty}=-g_{\xi}=\eta+c\left(\xi-\xi_{0}\right)+d\left(\eta-\eta_{0}\right) .
\end{aligned}
$$

At the critical point $u / u_{\infty} \rightarrow \xi_{0}$ and $v / u_{\infty} \rightarrow \eta_{0}$. The vorticity is

$$
\zeta=-\frac{\partial u}{\partial y}+\frac{\partial v}{\partial x}=-\frac{1}{t}(b-c)
$$

and the rate-of-strain tensor is

$$
\mathbf{S}=\left[\begin{array}{lr}
\frac{\partial u}{\partial x} & \frac{1}{2}\left(\frac{\partial u}{\partial y}+\frac{\partial v}{\partial x}\right) \\
\frac{1}{2}\left(\frac{\partial u}{\partial y}+\frac{\partial v}{\partial x}\right) & \frac{\partial v}{\partial y}
\end{array}\right]=\frac{1}{t}\left[\begin{array}{lr}
(1+a) & \frac{1}{2}(b+c) \\
\frac{1}{2}(b+c) & (1+d)
\end{array}\right]
$$

In the neighbourhood of a saddle, the eigenvectors of $\mathbf{A}$ are directed along the separatrices and have slopes

$$
m_{1,2}=\left(\lambda_{1,2}-a\right) / b=c /\left(\lambda_{1,2}-d\right),
$$

where $\lambda_{1}$ and $\lambda_{2}$ are the eigenvalues of $\mathbf{A}$. For $a+d=-2$, i.e. for $\partial u / \partial x+\partial v / \partial y=0$, the eigenvalues become

$$
\lambda_{1}=-1-(1-q)^{\frac{1}{2}}, \quad \lambda_{2}=-1+(1-q)^{\frac{1}{2}} .
$$

The included angle $\theta$ between the separatrices passing through the critical point is given by

$$
\cos \theta=\left(1+m_{1} m_{2}\right) /\left[\left(1+m_{1}^{2}\right)\left(+1 m_{2}^{2}\right)\right]^{\frac{1}{2}} .
$$

This equation can be rearranged to read

$$
\cos \theta=\frac{\frac{1}{2}(b-c)}{\left\{-(1+a)(1+d)+\left[\frac{1}{2}(b+c)\right]^{2}\right\}^{\frac{1}{2}}}=\frac{-\zeta}{2(-\operatorname{det} \mathbf{S})^{\frac{1}{2}}}
$$


which states that the angle between the separatrices through a saddle represents a balance between rotational motion (vorticity) and longitudinal straining motion (the root mean of the product of the principal strains). If $-\zeta / 2(-\operatorname{det} S)^{\frac{1}{2}}<1$, the fluid exhibits saddle behaviour. As this ratio increases through one, the angle $\theta$ passes through zero, the vorticity dominates and the fluid begins to exhibit focal behaviour. If the vorticity is zero, the angle is $90^{\circ}$.

The phase-plane method used in this paper could be extended to three dimensions. We let the velocity and pressure field depend only on $x / t, y / t$ and $z / t$ as follows:

$$
\begin{aligned}
u=u(x / t, y / t, z / t)=u(\xi, \eta, \sigma), & v=v(x / t, y / t, z / t)=v(\xi, \eta, \sigma), \\
w=w(x / t, y / t, z / t)=w(\xi, \eta, \sigma), & p=p(x / t, y / t, z / t)=p(\xi, \eta, \sigma) .
\end{aligned}
$$

Then Euler's equation becomes

$$
\begin{aligned}
& (u-\xi) \frac{\partial u}{\partial \xi}+(v-\eta) \frac{\partial u}{\partial \eta}+(w-\sigma) \frac{\partial u}{\partial \sigma}=-\frac{1}{\rho} \frac{\partial p}{\partial \xi} \\
& (u-\xi) \frac{\partial v}{\partial \xi}+(v-\eta) \frac{\partial v}{\partial \eta}+(w-\sigma) \frac{\partial v}{\partial \sigma}=-\frac{1}{\rho} \frac{\partial p}{\partial \eta} \\
& (u-\xi) \frac{\partial w}{\partial \xi}+(v-\eta) \frac{\partial w}{\partial \eta}+(w-\sigma) \frac{\partial w}{\partial \sigma}=-\frac{1}{\rho} \frac{\partial p}{\partial \sigma}
\end{aligned}
$$

and the particle-path equations become $(\tau=\ln t)$

$$
d \xi / d \tau=u-\xi, \quad d \eta / d \tau=v-\eta, \quad d \sigma / d \tau=w-\sigma .
$$

Measurements of all three velocity components in the spot could be used to map the complete three-dimensional entrainment pattern of the spot, in which case we should expect that the foci and saddles would extend to become horseshoe-shaped line singularities.

\section{REFERENCES}

Bevilaqua, P. M. \& Lykoudis, P.S. 1971 Mechanism of entrainment in turbulent wakes. A.I.A.A.J. 9, 1657-1659.

Brown, G. L. \& Roskro, A. 1974 On density effects and large structure in turbulent mixing layers. $J$. Fluid Mech. 64, 775-816.

Brown, G. L. \& Thomas, A. S. W. 1977 Large structure in a turbulent boundary layer. Phys. Fluids Suppl. 20, S243-S252.

CANTwELI, B. J. 1978 Similarity transformations for the two-dimensional, unsteady, streamfunction equation. $J$. Fluid Mech. 85, 257-271.

Coles, D. 1962 The turbulent boundary layer in a compressible fluid. Rand Corp. Rep. R-403-PR, appendix A.

CoLEs, D. \& BARker, S. J. 1975 Some remarks on a synthetic turbulent boundary layer. In Turbulent Mixing in Nonreactive and Reactive Flows (ed. S. N. B. Murthy), pp. 285-292. Plenum.

Corrsin, S. \& Kistuer, A. L. 1954 The free-stream boundaries of turbulent flows. N.A.C.A. Tech. Note no. 3133. (See also N.A.C.A. Tech. Rep. no. 1244, 1955.)

Drmotakis, P. E. 1976 Single scattering particle laser-Doppler measurements of turbulence. $A G A R D$ Conf. Proc. 193, pp. 10.1-10.14.

Dimotakis, P. E. \& Lang, D. B. 1974 Single scattering particle laser-Doppler velocimetry. Bull. Am. Phys. Soc. II 19, 1145.

ELDER, J.W. 1960 An experimental investigation of turbulent spots and breakdown to turbulence. J. Fluid Mech. 9, 235-246. 
Emmons, H. W. 1951 The laminar-turbulent transition in a boundary layer. Part I. J. Aero. Sci. 18, 490-498.

Hama, F. R. 1962 Streaklines in perturbed shear flow. Phys. Fluids 5, 644-650.

HunT, J. C. R. \& MuluEfarn, P. J. 1973 Turbulent dispersion from sources near two-dimensional obstacles. J. Fluid Mech. 61, 245-274.

Kline, S. J., Reynolds, W. C., Sohraub, F. A. \& Rungtadler, P. W. 1967 The structure of turbulent boundary layers. J. Fluid Mech. 30, 741-773.

Kovaszenay, L. S. G. 1949 Technique for the optical measurement of turbulence in high speed flow. Proc. Heat Transfer Fluid Mech. Inst., Berkeley, pp. 211-222.

KREID, D. K. 1974 Laser-Doppler velocimeter measurements in non-uniform flow: error estimates. Appl. Optics 13, 1872-1881.

McLaughitn, D. K. \& Tredermav, W. G. 1973 Biasing correction for individual realization of laser anemometer measurements in turbulent flows. Phys. Fluids 16, 2082-2088.

MAYo, W. T. 1974 A discussion of limitations and extensions of power spectrum estimation with burst-counter LDV systems. Proc. 2nd Int. Workshop Laser Velocimetry, Purdue Univ. (ed. H. D. Thompson \& W. H. Stevenson), vol. 1, pp. 90-101.

MEYer, K. A. \& KLINE, S. J. 1961 A visual study of the flow model in the later stages of laminar-turbulent transition on a flat plate. Stanford Univ., Dept. Mech. Engng Rep. MD-7.

Perry, A. E. \& FAIRLIE, B. D. 1974 Critical points in flow patterns. Adv. in Geophys. B 18, 299-315.

Schubauer, G. B. \& Krebanofr, P. S. 1955 Contributions on the mechanics of boundarylayer transition. N.A.C.A. Tech. Note no. 3489. (See also N.A.C.A. Tech. Rep. no. 1289, 1956.)

Sмгтн, A. M. O. 1954 Improved solutions of the Falkner and Skan boundary-layer equation. Inst. Aero. Sci., S. M. F. Fund. paper FF. 10.

Turner, J. S. 1964 The flow into an expanding spherical vortex. J. Fluid Mech. 18, 195-208.

WYgnaNsKi, I., Haritonidis, J. H. \& Kaplan, R. E. 1978 On a Tollmien-Schlichting wave packet produced by a turbulent spot. Submitted to $J$. Fluid Mech.

Wygnanski, I., Sokolov, N. \& Friedman, D. 1976 On the turbulent 'spot' in a boundary layer undergoing transition. J. Fluid Mech. 78, 785-819. 


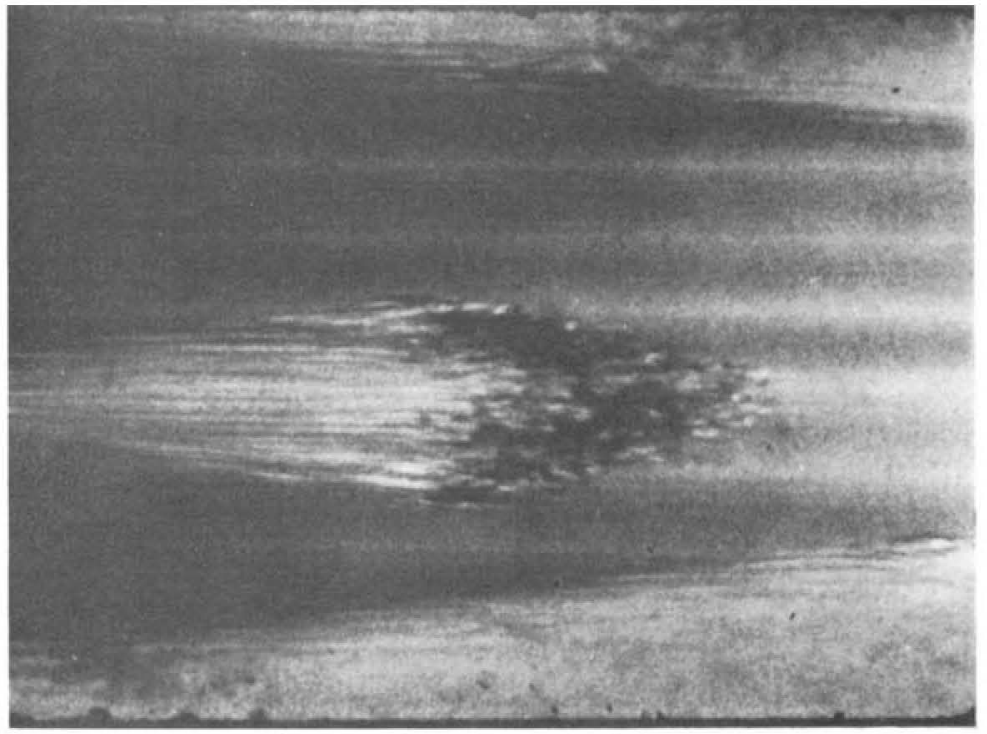

(a)

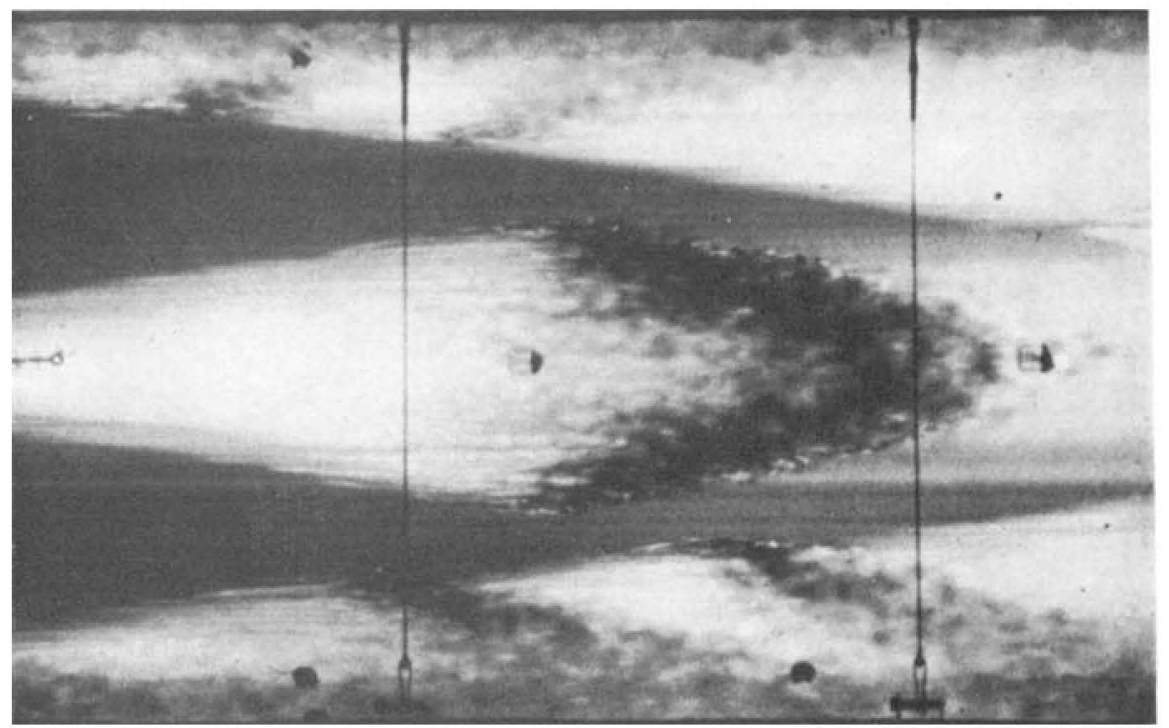

(b)

FigURe 4. Dye visualization of turbulent spots in water. (a) Spot at low Reynolds number, from flow studies by Coles \& I3arker (Coles, private communication); $U_{\infty}=32 \mathrm{~cm} / \mathrm{s}$. (b) Spot at relatively high Reynolds number, from present expcriments; $U_{\infty}=59 \mathrm{~cm} / \mathrm{s}$. Transverse lines in (b) are cables $30.5 \mathrm{~cm}$ apart. Note clear wake compared with $(a)$. 

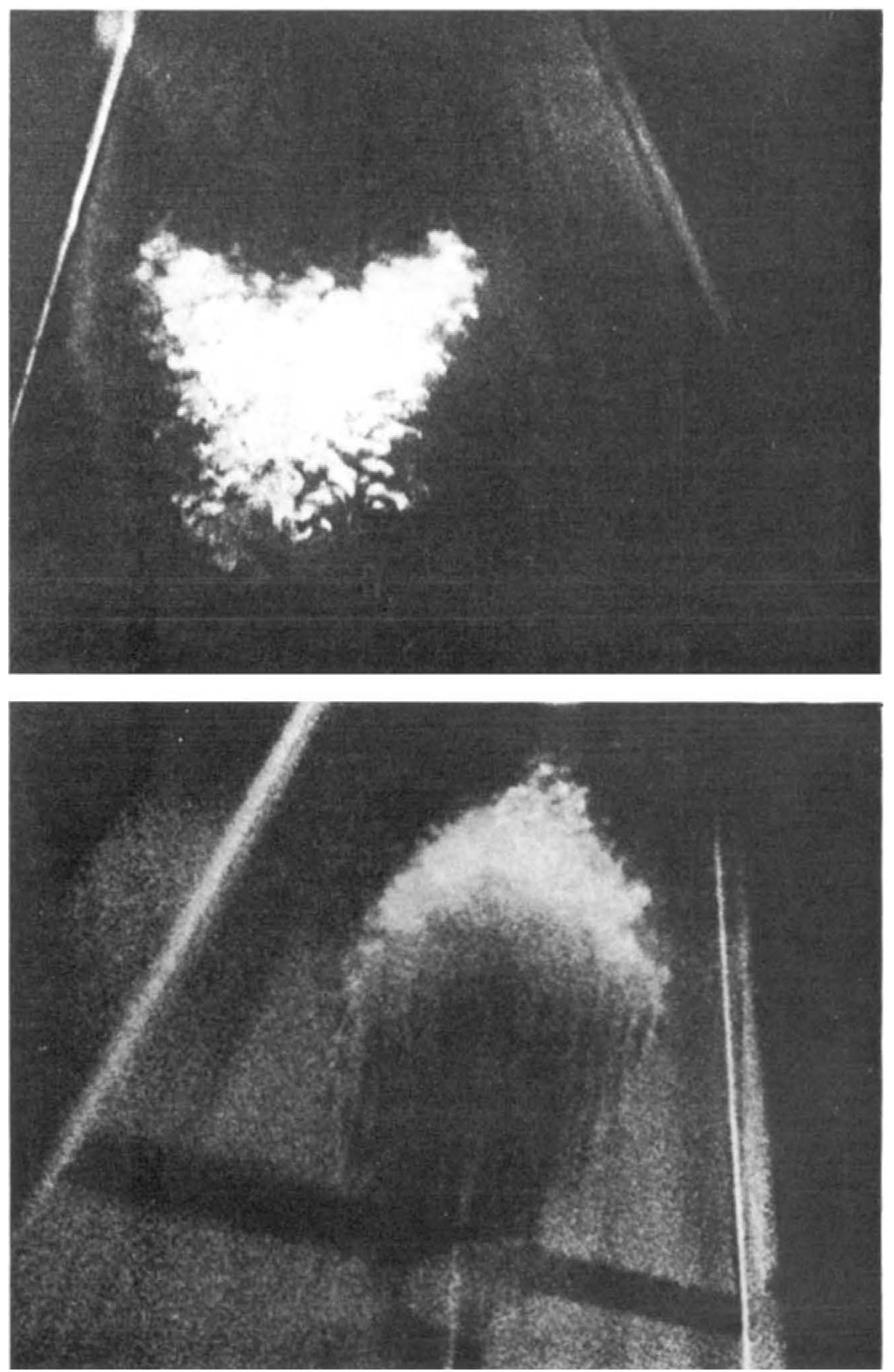

Frgure 5. Smoke visualization of turbulent spots in air (courtesy of M. R. Head). (a) Oblique view of spot approaching observer, with illumination by light sheet above laminar boundary layer. (b) Oblique view of spot receding from observer, with more diffuse illumination that in $(a)$. 

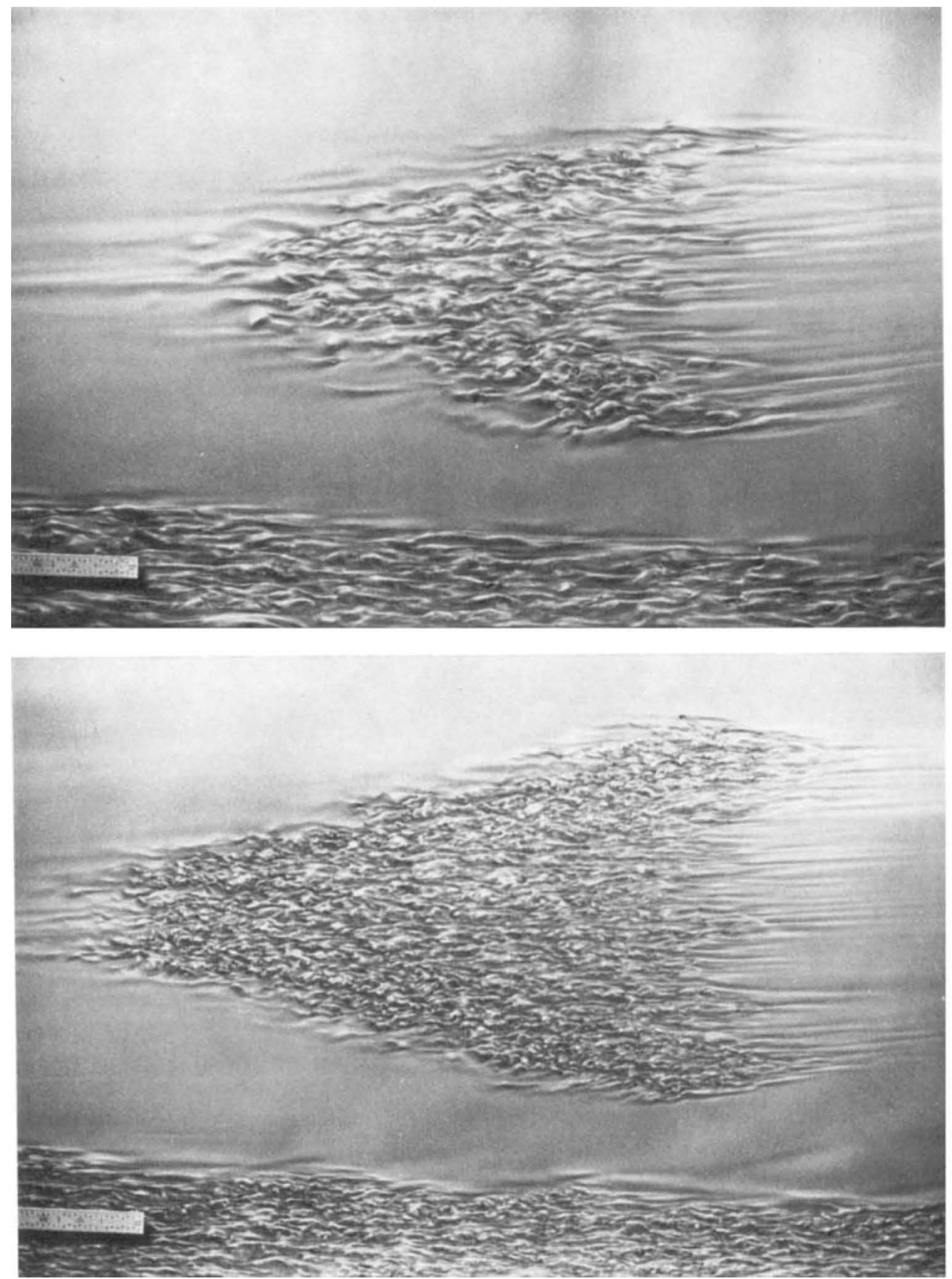

FiGURE 6. Aluminium visualization of sublayer streaks for turbulent spots in water. (a) L Low Reynolds number; $U_{\infty}=12 \mathrm{~cm} / \mathrm{s}$. (b) Higher Reynolds number; $U_{\infty}=23 \mathrm{~cm} / \mathrm{s}$. Note region of transvorse contamination from channel bottom. 

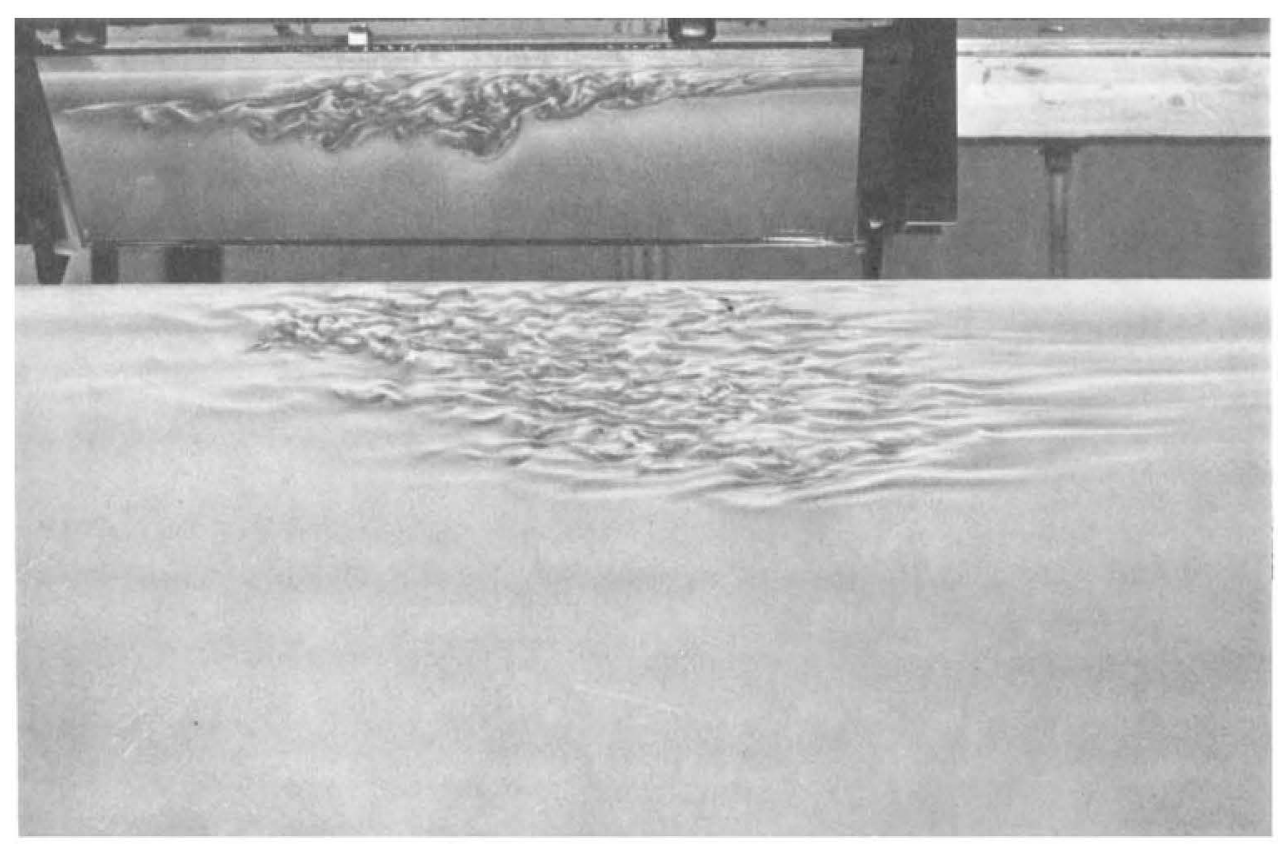

(a)

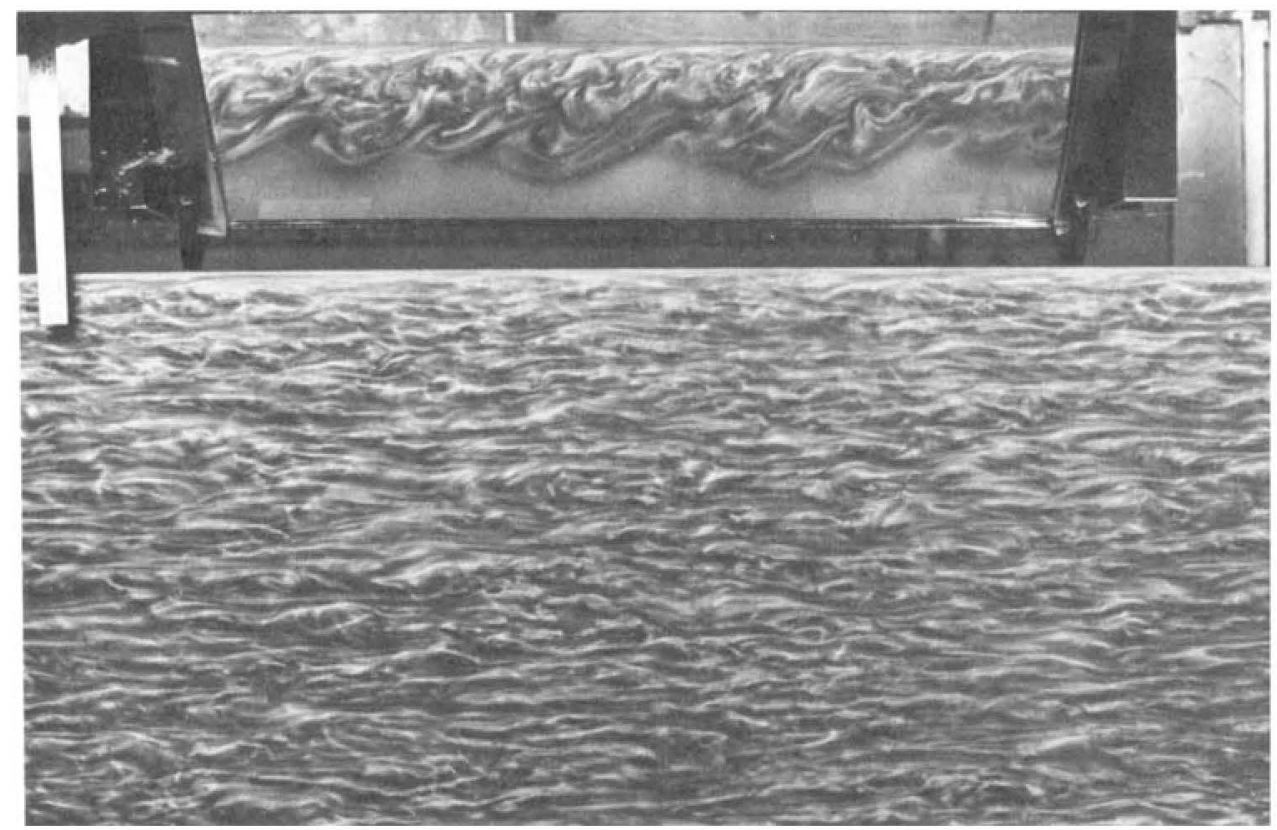

Figure 7. Aluminium visualization of sublayer streaks for half-spot and for boundary layor. (a) Half-spot at low Reynolds number; $U_{\infty}=12 \mathrm{~cm} / \mathrm{s}$. Mirror is used to show simultanoous view of free surface. (b) As in (a), but with fully turbulent boundary layer; $U_{\infty}=15 \mathrm{~cm} / \mathrm{s}$. 